Pacific

Journal of

Mathematics

\title{
ON FOURIER COEFFICIENTS OF CERTAIN RESIDUAL REPRESENTATIONS OF SYMPLECTIC GROUPS
}

DIHUA JIANG AND BAIYING LIU 


\title{
ON FOURIER COEFFICIENTS OF CERTAIN RESIDUAL REPRESENTATIONS OF SYMPLECTIC GROUPS
}

\author{
DIHUA JIANG AND BAIYING LIU
}

\begin{abstract}
In the theory of automorphic descents developed by Ginzburg, Rallis, and Soudry in The descent map from automorphic representations of GL(n) to classical groups (World Scientific, 2011), the structure of Fourier coefficients of the residual representations of certain special Eisenstein series plays an essential role. In a series of papers starting with Pacific J. Math. 264:1 (2013), 83-123, we have looked for more general residual representations, which may yield a more general theory of automorphic descents. We continue this program here, investigating the structure of Fourier coefficients of certain residual representations of symplectic groups, associated with certain interesting families of global Arthur parameters. The results partially confirm a conjecture proposed by Jiang in Contemp. Math. 614 (2014), 179-242 on relations between the global Arthur parameters and the structure of Fourier coefficients of the automorphic representations in the associated global Arthur packets. The results of this paper can also be regarded as a first step towards more general automorphic descents for symplectic groups, which will be considered in our future work.
\end{abstract}

\section{Introduction}

Let $\mathrm{Sp}_{2 n}$ be the symplectic group with symplectic form

$$
\left(\begin{array}{cc}
0 & v_{n} \\
-v_{n} & 0
\end{array}\right)
$$

where $v_{n}$ is an $n \times n$ matrix with $1 \mathrm{~s}$ on the second diagonal and 0 s elsewhere. Fix a Borel subgroup $B=T U$ of $\mathrm{Sp}_{2 n}$, where the maximal torus $T$ consists of elements of the form

$$
\operatorname{diag}\left(t_{1}, \ldots, t_{n} ; t_{n}^{-1}, \ldots, t_{1}^{-1}\right)
$$

The research of Jiang is supported in part by the NSF Grants DMS-1301567, and the research of Liu is supported in part by NSF Grants DMS-1302122, and in part by a postdoc research fund from Department of Mathematics, University of Utah.

MSC2010: primary 11F70, 22E55; secondary 11F30.

Keywords: Arthur parameters, Fourier coefficients, unipotent orbits, automorphic forms. 
and the unipotent radical $U$ consists of all upper unipotent matrices in $\mathrm{Sp}_{2 n}$. Let $F$ be a number field and $\mathbb{A}$ be the ring of adeles of $F$.

The structure of Fourier coefficients for the residual representations of $\operatorname{Sp}_{4 n}(\mathbb{A})$, with cuspidal support $\left(\mathrm{GL}_{2 n}, \tau\right)$, played an indispensable role in the theory of automorphic descent from $\mathrm{GL}_{2 n}$ to the metaplectic double cover of $\mathrm{Sp}_{2 n}$ by Ginzburg, Rallis, and Soudry in [Ginzburg et al. 2011]. As tested in a special case in our recent work joint with Xu and Zhang in [Jiang et al. 2015], we expected the residual representations investigated in [Jiang et al. 2013] may play important roles in extending the theory of automorphic descent in [Ginzburg et al. 2011] to a more general setting. In this paper, we take certain interesting families of residual representations of $\mathrm{Sp}_{2 n}(\mathbb{A})$ obtained in [Jiang et al. 2013] and study the structure of their Fourier coefficients associated to nilpotent orbits as described in [Jiang 2014]. On one hand, the results of this paper partially confirm a conjecture proposed by the first named author in [loc. cit.] on relations between the global Arthur parameters and the structure of Fourier coefficients of the automorphic representations in the corresponding global Arthur packets. On the other hand, these results are preliminary steps towards the theory of more general automorphic descents for symplectic groups, which will be considered in our future work.

We first recall the global Arthur parameters for $\mathrm{Sp}_{2 n}$ and the discrete spectrum, and the conjecture made in [loc. cit.]. Then we recall what has been proved about this conjecture before this current paper, in particular the results obtained in [Jiang and Liu 2015a]. Finally we describe more explicitly the objective of this paper. The main results will be precisely stated in Section 2 .

1A. Arthur parameters and the discrete spectrum. Let $F$ be a number field and $A$ be the ring of adeles of $F$. Recall that the dual group of $G_{n}=\mathrm{Sp}_{2 n}$ is $\mathrm{SO}_{2 n+1}(\mathbb{C})$. The set of global Arthur parameters for the discrete spectrum of the space of all square-integrable automorphic functions on $\operatorname{Sp}_{2 n}(\mathbb{A})$ is denoted by $\widetilde{\Psi}_{2}\left(\operatorname{Sp}_{2 n}\right)$, following the notation in [Arthur 2013]. The elements of $\widetilde{\Psi}_{2}\left(\mathrm{Sp}_{2 n}\right)$ are of the form

$$
\psi:=\psi_{1} \boxplus \psi_{2} \boxplus \cdots \boxplus \psi_{r},
$$

where $\psi_{i}$ are pairwise distinct simple global Arthur parameters of orthogonal type. A simple global Arthur parameter is formally given by $(\tau, b)$ with an integer $b \geq 1$, and with $\tau \in \mathcal{A}_{\text {cusp }}(a)$ being an irreducible unitary cuspidal automorphic representation of $\mathrm{GL}_{a}(\mathbb{A})$.

In (1-1), one has that $\psi_{i}=\left(\tau_{i}, b_{i}\right)$ with $\tau_{i} \in \mathcal{A}_{\text {cusp }}\left(a_{i}\right), 2 n+1=\sum_{i=1}^{r} a_{i} b_{i}$, and $\prod_{i} \omega_{\tau_{i}}^{b_{i}}=1$ (the condition on the central character of the parameter), following [Arthur 2013, Section 1.4]. In order for all the $\psi_{i}$ to be of orthogonal type, the simple parameters $\psi_{i}=\left(\tau_{i}, b_{i}\right)$ for $i=1,2, \ldots, r$ satisfy the following parity condition: if $\tau_{i}$ is of symplectic type (i.e., $L\left(s, \tau_{i}, \bigwedge^{2}\right)$ has a pole at $s=1$ ), then 
$b_{i}$ is even; and if $\tau_{i}$ is of orthogonal type (i.e., $L\left(s, \tau_{i}, \mathrm{Sym}^{2}\right)$ has a pole at $s=1$ ), then $b_{i}$ is odd. A global Arthur parameter $\psi=\boxplus_{i=1}^{r}\left(\tau_{i}, b_{i}\right)$ is called generic if $b_{i}=1$ for all $1 \leq i \leq r$.

Theorem 1.1 [Arthur 2013, Theorem 1.5.2]. For each global Arthur parameter $\psi \in \widetilde{\Psi}_{2}\left(\operatorname{Sp}_{2 n}\right)$, there exists a global Arthur packet $\widetilde{\Pi}_{\psi}$. The discrete spectrum of $\mathrm{Sp}_{2 n}(\mathbb{A})$ has the following decomposition

$$
L_{\text {disc }}^{2}\left(\operatorname{Sp}_{2 n}(F) \backslash \operatorname{Sp}_{2 n}(\mathbb{A})\right) \cong \bigoplus_{\substack{\psi \in \widetilde{\Psi}_{2}\left(\operatorname{Sp}_{2 n}\right) \\ \pi \in \widetilde{\Pi}_{\psi}\left(\epsilon_{\psi}\right)}} \pi,
$$

where $\widetilde{\Pi}_{\psi}\left(\epsilon_{\psi}\right)$ denotes the subset of $\widetilde{\Pi}_{\psi}$ consisting of members which occur in the discrete spectrum of $\mathrm{Sp}_{2 n}(\mathbb{A})$.

1B. A conjecture on the Fourier coefficients. We will use the notation in [Jiang and Liu 2015c; 2015a] freely. Following [Jiang and Liu 2015c, Section 2], for a symplectic partition $p$ of $2 n$, or equivalently each $F$-stable unipotent orbit $\mathcal{O}_{p}$, via the standard $\mathfrak{s l}_{2}(F)$-triple, one may construct an $F$-unipotent subgroup $V_{p, 2}$. In this case, the $F$-rational unipotent orbits in the $F$-stable unipotent orbit $\mathcal{O}_{p}$ are parametrized by a datum $\underline{a}$ (see [loc. cit.] for details), which defines a character $\psi_{p, \underline{a}}$ of $V_{p, 2}(\mathbb{A})$. This character $\psi_{p, a}$ is automorphic in the sense that it is trivial on $V_{p, 2}(F)$. The $\psi_{p, \underline{a}}$-Fourier coefficient of an automorphic form $\varphi$ on $\operatorname{Sp}_{2 n}(\mathbb{A})$ is defined by

$$
\varphi^{\psi_{\underline{p}, \underline{a}}}(g):=\int_{V_{\underline{p}, 2}(F) \backslash V_{\underline{p}, 2}(\mathrm{~A})} \varphi(v g) \psi_{\underline{p}, \underline{a}}(v)^{-1} d v .
$$

We say that an irreducible automorphic representation $\pi$ of $\operatorname{Sp}_{2 n}(\mathbb{A})$ has a nonzero $\psi_{\underline{p}, \underline{a}}$-Fourier coefficient or a nonzero Fourier coefficient attached to a (symplectic) partition $\underline{p}$ if there exists an automorphic form $\varphi$ in the space of $\pi$ with a nonzero $\psi_{\underline{p}, \underline{a}}$-Fourier coefficient $\varphi^{\psi_{\underline{p}}, \underline{a}}(\mathrm{~g})$, for some choice of $\underline{a}$. For any irreducible automorphic representation $\pi$ of $\operatorname{Sp}_{2 n}(\mathbb{A})$, as in [Jiang 2014], we define $\mathfrak{p}^{m}(\pi)$ (which corresponds to $\mathfrak{n}^{m}(\pi)$ in the notation of [loc. cit.]) to be the set of all symplectic partitions $\underline{p}$ with the properties that $\pi$ has a nonzero $\psi_{p, \underline{a}}$-Fourier coefficient for some choice of $\underline{a}$, but for any $\underline{p}^{\prime}>\underline{p}$ (with the natural ordering of partitions), $\pi$ has no nonzero Fourier coefficients attached to $\underline{p}^{\prime}$. It is generally believed (and may be called a conjecture) that the set $\mathfrak{p}^{m}(\pi)$ contains only one partition for any irreducible automorphic representation $\pi$ (or locally for any irreducible admissible representation $\pi$ ). We refer to [Jiang and Liu 2015b, Section 3], in particular Conjecture 3.1, for more detailed discussions on this issue.

As in [Jiang 2014], $\widetilde{\Pi}_{\psi}\left(\epsilon_{\psi}\right)$ is called the automorphic $L^{2}$-packet attached to the global Arthur parameter $\psi$. For each $\psi$ of the form in (1-1), let $p(\psi)=$ $\left[\left(b_{1}\right)^{\left(a_{1}\right)} \cdots\left(b_{r}\right)^{\left(a_{r}\right)}\right]$ be a partition of $2 n+1$ attached to the global Arthur parameter 
$\psi$, following the discussion in [op. cit., Section 4]. For $\pi \in \widetilde{\Pi}_{\psi}\left(\epsilon_{\psi}\right)$, the structure of the global Arthur parameter $\psi$ deduces constraints on the structure of $\mathfrak{p}^{m}(\pi)$, which are given by the following conjecture.

Conjecture 1.2 [Jiang 2014, Conjecture 4.2]. For any $\psi \in \widetilde{\Psi}_{2}\left(\operatorname{Sp}_{2 n}\right)$, let $\widetilde{\Pi}_{\psi}\left(\epsilon_{\psi}\right)$ be the automorphic $L^{2}$-packet attached to $\psi$. Then the following hold.

(1) Any symplectic partition $p$ of $2 n$ satisfying $p>\eta_{\mathfrak{g}^{\vee}, \mathfrak{g}}(p(\psi))$ does not belong to $\mathfrak{p}^{m}(\pi)$ for any $\pi \in \widetilde{\Pi}_{\psi}\left(\epsilon_{\psi}\right)$.

(2) For every $\pi \in \widetilde{\Pi}_{\psi}\left(\epsilon_{\psi}\right)$, every partition $\underline{p} \in \mathfrak{p}^{m}(\pi)$ has the property that $\underline{p} \leq \eta_{\mathfrak{g} \vee}, \mathfrak{g}(\underline{p}(\psi))$.

(3) There exists at least one member $\pi \in \widetilde{\Pi}_{\psi}\left(\epsilon_{\psi}\right)$ having the property that $\eta_{\mathfrak{g} \vee}, \mathfrak{g}(\underline{p}(\psi)) \in \mathfrak{p}^{m}(\pi)$.

Here $\eta_{\mathfrak{g} \vee}, \mathfrak{g}$ denotes the Barbasch-Vogan duality map (see Definition 2.2) from the partitions for $\mathfrak{s o}_{2 n+1}(\mathbb{C})$ to the partitions for $\mathfrak{s p}_{2 n}(\mathbb{C})$.

We remark that part (2) is stronger than part (1) in Conjecture 1.2. More related discussions can be found in [Jiang and Liu 2015b].

There has been progress toward the proof of Conjecture 1.2. When the global Arthur parameter $\psi=\boxplus_{i=1}^{r}\left(\tau_{i}, 1\right)$ is generic, in Conjecture 1.2, part (1) is trivial, part (2) is automatic, and part (3) of Conjecture 1.2 can be viewed as the global version of the Shahidi conjecture, namely, any global tempered $L$-packet has a generic member. This can be proved following the theory of automorphic descent developed by Ginzburg, Rallis, and Soudry [Ginzburg et al. 2011] and the endoscopy classification of Arthur [2013]. We refer to [Jiang and Liu 2015b, Section 3.1], in particular Theorem 3.3, for more precise discussion on this issue. Hence Conjecture 1.2 holds for all generic global Arthur parameters, and those $\pi$ satisfying part (3) are generic cuspidal representations.

For Arthur parameters of form $\psi=(\tau, b) \boxplus\left(1_{\mathrm{GL}_{1}(\mathbb{A})}, 1\right)$, where $\tau$ is an irreducible cuspidal representation of $\mathrm{GL}_{2 k}(\mathbb{A})$ and is of symplectic type, and $b$ is even, one has that $p(\psi)=\left[b^{(2 k)} 1\right]$. In this case, part (3) of Conjecture 1.2 has been proved by Liu in [2013a], where it is also shown that $\mathfrak{p}^{m}(\pi)$ contains only one partition in this particular case.

For a general global Arthur parameter $\psi$, part (1) of Conjecture 1.2 is completely proved in [Jiang and Liu 2015a]. We remark that if we assume that $\mathfrak{p}^{m}(\pi)$ contains only one partition, then part (2) of Conjecture 1.2 essentially follows from parts (1) and (3) of Conjecture 1.2 plus certain local constraints at unramified local places as discussed in [loc. cit.]. We omit the details here. However, without knowing that the set $\mathfrak{p}^{m}(\pi)$ contains only one partition, part (2) of Conjecture 1.2 is also settled in [loc. cit.] partially; namely, any symplectic partition $p$ of $2 n$, for which $\underline{p}>\eta_{\mathfrak{g} \vee}, \mathfrak{g}(\underline{p}(\psi))$ under the lexicographical ordering, does not belong to $\mathfrak{p}^{m}(\pi)$ 
for any $\pi \in \widetilde{\Pi}_{\psi}\left(\epsilon_{\psi}\right)$. We refer to [Jiang 2014, Section 4] and also [Jiang and Liu 2015b] for more discussion on this conjecture and related topics.

1C. The objective of this paper. In this section, we begin to investigate part (3) of Conjecture 1.2. This means that we have to construct or determine a particular member in a given automorphic $L^{2}$-packet $\widetilde{\Pi}_{\psi}\left(\epsilon_{\psi}\right)$ attached to a general global Arthur parameter $\psi$, whose Fourier coefficients achieve the partition $\eta_{\mathfrak{g} \vee}, \mathfrak{g}(p(\psi))$. Such members should be the distinguished members in $\widetilde{\Pi}_{\psi}\left(\epsilon_{\psi}\right)$, following the Whittaker normalization in the sense of Arthur [2013] for global generic Arthur parameters. For general nongeneric global Arthur parameters, the distinguished members in $\widetilde{\Pi}_{\psi}\left(\epsilon_{\psi}\right)$ can be certain residual representations determined by $\psi$ as conjectured by Mœglin [2008; 2011], or certain cuspidal automorphic representations, which may be explicitly constructed through the framework of endoscopy correspondences as outlined in [Jiang 2014]. Due to the different nature of the two construction methods, we are going to treat them separately, in order to prove part (3) of Conjecture 1.2.

As explained in [Jiang and Liu 2015b], when the distinguished members $\pi$ in a given $\widetilde{\Pi}_{\psi}\left(\epsilon_{\psi}\right)$ are residual representations, they can be constructed explicitly from the given cuspidal data. In this case, our method is to establish the nonvanishing of the Fourier coefficients of those $\pi$ associated to the partition $\eta_{\mathfrak{g} \vee}, \mathfrak{g}(p(\psi))$, in terms of the nonvanishing condition (Fourier coefficients or periods) on the construction data that is also defined by the given nongeneric global Arthur parameter $\psi$. Hence, such a method can be regarded as a natural extension of the wellknown Langlands-Shahidi method from generic Eisenstein series [Shahidi 2010] to nongeneric Eisenstein series, and in particular to the singularity of Eisenstein series, i.e., the residues of Eisenstein series. On the other hand, this method can also be regarded as an extension of the automorphic descent method of Ginzburg-RallisSoudry for particular residual representations [Ginzburg et al. 2011] to general residual representations.

In this paper, we are going to test our method for these nongeneric global Arthur parameters $\psi$, whose automorphic $L^{2}$-packets $\widetilde{\Pi}_{\psi}\left(\epsilon_{\psi}\right)$ contain the residual representations that are completely determined in our previous work joint with Zhang [Jiang et al. 2013]. Those nongeneric global Arthur parameters of $\operatorname{Sp}_{2 n}(\mathbb{A})$ are of the following form

$$
\psi=\left(\tau_{1}, b_{1}\right) \boxplus \underset{i=2}{r}\left(\tau_{i}, 1\right), \quad \text { with } b_{1}>1,
$$

which has three cases, depending on the symmetry of $\tau_{1}$ and the relationship between $\tau_{1}$ and $\tau_{i}$ for $i=2,3, \ldots, r$. In each case, $b \geq 1$.

Case I: $\quad \psi=(\tau, 2 b+1) \boxplus \boxplus_{i=2}^{r}\left(\tau_{i}, 1\right), \quad$ where $\tau \not \equiv \tau_{i}$ for any $2 \leq i \leq r$. 
Case II: $\psi=(\tau, 2 b+1) \boxplus(\tau, 1) \boxplus \boxplus_{i=3}^{r}\left(\tau_{i}, 1\right), \quad$ where $\tau \not \tau_{i}$ for any $3 \leq i \leq r$. Case III: $\psi=(\tau, 2 b) \boxplus \boxplus_{i=2}^{r}\left(\tau_{i}, 1\right)$.

For $\psi \in \widetilde{\Psi}_{2}\left(\mathrm{Sp}_{2 n}\right), \tau \in \mathcal{A}_{\text {cusp }}\left(\mathrm{GL}_{a}\right)$ is of orthogonal type in Case I and Case II, and of symplectic type in Case III. Of course, the remaining $\tau_{i}$ are of orthogonal type in all three cases.

When $\tau$ is of orthogonal type, i.e., in both Case I and Case II, the corresponding residual representations given in [Jiang et al. 2013] must be nonzero. In this paper, we prove part (3) of Conjecture 1.2 in those two cases, and refer to Section 2 for more details.

When $\tau$ is of symplectic type and $r \geq 2$, the relation between $\tau$ and $\tau_{i}$, for $i=2,3, \ldots, r$, is governed by the corresponding Gan-Gross-Prasad conjecture [Gan et al. 2012], which controls the structure of the automorphic $L^{2}$-packet $\widetilde{\Pi}_{\psi}\left(\epsilon_{\psi}\right)$. We prove part (3) of Conjecture 1.2 for Case III when $\widetilde{\Pi}_{\psi}\left(\epsilon_{\psi}\right)$ contains residual representations. While the automorphic $L^{2}$-packet $\widetilde{\Pi}_{\psi}\left(\epsilon_{\psi}\right)$ does not contain any residual representation, the situation is more involved, and will be left for a separate treatment in our future work. We discuss with more details in Section 2.

We will state the main results more explicitly in Section 2. After recalling a technical lemma from [Jiang and Liu 2015b] in Section 3, we are ready to treat Case I in both Sections 4 and 5. Case II is treated in Section 6. The final section is devoted to Case III. One may find more detailed description of the arguments and methods used in the proof of those cases in each relevant section.

\section{The main results}

After introducing more notation and basic facts about the discrete spectrum and Fourier coefficients attached to partitions, we will state the main results explicitly for each case.

Throughout the paper, we let $P_{r}^{2 n}=M_{r}^{2 n} N_{r}^{2 n}$ (with $1 \leq r \leq n$ ) be the standard parabolic subgroup of $\mathrm{Sp}_{2 n}$ with Levi part $M_{r}^{2 n}$ isomorphic to $\mathrm{GL}_{r} \times \mathrm{Sp}_{2 n-2 r}$ and unipotent radical $N_{r}^{2 n}$. Also let $\tilde{P}_{r}^{2 n}(\mathbb{A})=\tilde{M}_{r}^{2 n}(\mathbb{A}) N_{r}^{2 n}(\mathbb{A})$ be the preimage of $P_{r}^{2 n}(\mathbb{A})$ in $\widetilde{\operatorname{Sp}}_{2 n}(\mathbb{A})$ (the superscript $2 n$ may be dropped when there is no confusion). The description of the three cases was briefly given in [Jiang and Liu 2015b]. Here are the details.

2A. Case I. $\psi \in \widetilde{\Psi}_{2}\left(\mathrm{Sp}_{2 n}\right)$ is written as

$$
\psi=(\tau, 2 b+1) \boxplus \underset{i=2}{r}\left(\tau_{i}, 1\right),
$$

where $b \geq 1$ and $\tau \not \tau_{i}$ for any $2 \leq i \leq r$. Assume $\tau \in \mathcal{A}_{\text {cusp }}\left(\mathrm{GL}_{a}\right)$ has central character $\omega_{\tau}$, and $\tau_{i} \in \mathcal{A}_{\text {cusp }}\left(\mathrm{GL}_{a_{i}}\right)$ has central character $\omega_{\tau_{i}}$ for $2 \leq i \leq r$. Following 
the definition of $\widetilde{\Psi}_{2}\left(\mathrm{Sp}_{2 n}\right)$, one must have that $2 n+1=a(2 b+1)+\sum_{i=2}^{r} a_{i}$, and $\omega_{\tau}^{2 b+1} \cdot \prod_{i=2}^{r} \omega_{\tau_{i}}=1$. Consider the isobaric representation $\pi=\tau \boxplus \tau_{2} \boxplus \cdots \boxplus \tau_{r}$ of $\mathrm{GL}_{2 m+1}(\mathbb{A})$, where $2 m+1=a+\sum_{i=2}^{r} a_{i}=2 n+1-2 a b$. It follows that $\pi$ has central character $\omega_{\pi}=\omega_{\tau} \cdot \prod_{i=2}^{r} \omega_{\tau_{i}}=1$ and $a \leq 2 m+1=2 n+1-2 a b$.

By [Ginzburg et al. 2011, Theorem 3.1], $\pi$ descends to an irreducible generic cuspidal representation $\sigma$ of $\operatorname{Sp}_{2 n-2 a b}(\mathbb{A})$, which has the functorial transfer back to $\pi$. As remarked before, this is part (3) of Conjecture 1.2 for the generic global Arthur parameter

$$
\psi_{\pi}=(\tau, 1) \boxplus\left(\tau_{2}, 1\right) \boxplus \cdots \boxplus\left(\tau_{r}, 1\right) .
$$

Hence $L(s, \tau \times \sigma)$ has a (simple) pole at $s=1$.

Let $\Delta(\tau, b)$ be the Speh residual representation in the discrete spectrum of $\mathrm{GL}_{a b}(\mathbb{A})$; see [Mœglin and Waldspurger 1989], or [Jiang et al. 2013, Section 1.2]. For any automorphic form

$$
\phi \in \mathcal{A}\left(N_{a b}(\mathbb{A}) M_{a b}(F) \backslash \operatorname{Sp}_{2 a b+2 m}(\mathbb{A})\right)_{\Delta(\tau, b) \otimes \sigma},
$$

following [Langlands 1976; Mœglin and Waldspurger 1995], one has a residual Eisenstein series

$$
E(\phi, s)(g)=E\left(g, \phi_{\Delta(\tau, b) \otimes \sigma}, s\right) .
$$

We refer to [Jiang et al. 2013] for particular details about this family of Eisenstein series. In particular, it is proved in [Jiang et al. 2013] that $E(\phi, s)(g)$ has a simple pole at $(b+1) / 2$, which is the right-most one. We denote by $\mathcal{E}(g, \phi)$ the residue, which is square-integrable. They generate the residual representation $\mathcal{E}_{\Delta(\tau, b) \otimes \sigma}$ of $\mathrm{Sp}_{2 n}(\mathbb{A})$. Following [Jiang et al. 2013, Section 6.2], the global Arthur parameter of this nonzero square-integrable automorphic representation $\mathcal{E}_{\Delta(\tau, b) \otimes \sigma}$ is exactly $\psi=(\tau, 2 b+1) \boxplus \boxplus_{i=2}^{r}\left(\tau_{i}, 1\right)$ as in (2-1). We prove part (3) of Conjecture 1.2 for Case I.

Theorem 2.1. For any global Arthur parameter of the form

$$
\psi=(\tau, 2 b+1) \boxplus \bigoplus_{i=2}^{r}\left(\tau_{i}, 1\right)
$$

with $b \geq 1$ and $\tau \neq \tau_{i}$ for any $2 \leq i \leq r$, the residual representation $\mathcal{E}_{\Delta(\tau, b) \otimes \sigma}$ has a nonzero Fourier coefficient attached to the Barbasch-Vogan duality

$$
\eta_{\mathfrak{s o}_{2 n+1}, \mathfrak{s p}_{2 n}}(\underline{p}(\psi))
$$

of the partition $\underline{p}(\psi)$ associated to $\left(\psi, \mathrm{SO}_{2 n+1}(\mathbb{C})\right)$.

In order to prove Theorem 2.1, we have to precisely figure out the partition $\eta_{\mathfrak{s o}_{2 n+1}, \mathfrak{s p}_{2 n}}(\underline{p}(\psi))$. We recall 
Definition 2.2. Given any partition $p q=\left[q_{1} q_{2} \cdots q_{r}\right]$ for $\mathfrak{s o}_{2 n+1}(\mathbb{C})$ satisfying $q_{1} \geq q_{2} \geq \cdots \geq q_{r}>0$, whose even parts occur with even multiplicity, let $q^{-}=$ $\left[q_{1} q_{2} \cdots q_{r-1}\left(q_{r}-1\right)\right]$. Then the Barbasch-Vogan duality $\eta_{\mathfrak{s o}_{2 n+1}, \mathfrak{s p}_{2 n}}$, following [Barbasch and Vogan 1985, Definition A1; Achar 2003, Section 3.5], is defined by

$$
\eta_{\mathfrak{s o}_{2 n+1}, \mathfrak{s p}_{2 n}}(\underline{q}):=\left(\left(\underline{q}^{-}\right)_{\operatorname{Sp}_{2 n}}\right)^{t},
$$

where $\left(q^{-}\right)_{\mathrm{Sp}_{2 n}}$ is the $\mathrm{Sp}_{2 n}$-collapse of $\underline{q}^{-}$, which is the biggest special symplectic partition which is smaller than $q^{-}$.

Following [Jiang 2014, Section 4], $p(\psi)=\left[(2 b+1)^{a}(1)^{2 m+1-a}\right]$. As calculated in [Jiang and Liu 2015b], when $a=2 m+1$, by Definition 2.2,

$$
\eta_{\mathfrak{s o}_{2 n+1}, \mathfrak{s p}_{2 n}}(\underline{p}(\psi))=\left[(a)^{2 b}(2 m)\right] ;
$$

when $a \leq 2 m$ and $a$ is even,

$$
\eta_{\mathfrak{s o}_{2 n+1}, \mathfrak{s p}_{2 n}}(\underline{p}(\psi))=\left[(2 m)(a)^{2 b}\right] ;
$$

and finally, when $a \leq 2 m$ and $a$ is odd,

$$
\eta_{\mathfrak{s o}_{2 n+1}, \mathfrak{s p}_{2 n}}(\underline{p}(\psi))=\left[(2 m)(a+1)(a)^{2 b-2}(a-1)\right] .
$$

The proof of Theorem 2.1 goes as follows. Given a symplectic partition $\underline{p}$ of $2 n$ (that is, where odd parts occur with even multiplicities), denote by $\underline{p}^{\mathrm{Sp}_{2 n}}$ the $\mathrm{Sp}_{2 n}$-expansion of $\underline{p}$, which is the smallest special symplectic partition that is bigger than $\underline{p}$. In [Jiang and Liu 2015c], we proved the following theorem which provides a crucial reduction in the proof of Theorem 2.1.

Theorem 2.3 [Jiang and Liu 2015c, Theorem 4.1]. Let $\pi$ be an irreducible automorphic representation of $\mathrm{Sp}_{2 n}(\mathbb{A})$. If $\pi$ has a nonzero Fourier coefficient attached to a nonspecial symplectic partition $p$ of $2 n$, then $\pi$ must have a nonzero Fourier coefficient attached to $\underline{p}^{\mathrm{Sp}_{2 n}}$, the $\mathrm{Sp}_{2 n}$-expansion of the partition $\underline{p}$.

If $a \leq 2 m$ and $a$ is odd, by [Collingwood and McGovern 1993, Lemma 6.3.9],

$$
\left[(2 m)(a+1)(a)^{2 b-2}(a-1)\right]=\left[(2 m)(a)^{2 b}\right]^{\mathrm{Sp}_{2 n}} .
$$

Hence it suffices to prove the following theorem.

Theorem 2.4. With notation above, the following hold.

(1) If $a=2 m+1$, then $\mathcal{E}_{\Delta(\tau, b) \otimes \sigma}$ has a nonzero Fourier coefficient attached to $\left[(a)^{2 b}(2 m)\right]$.

(2) If $a \leq 2 m$, then $\mathcal{E}_{\Delta(\tau, b) \otimes \sigma}$ has a nonzero Fourier coefficient attached to $\left[(2 m)(a)^{2 b}\right]$.

Parts (1) and (2) of Theorem 2.4 will be proved in Sections 4 and 5, respectively. 
2B. Case II. $\psi \in \widetilde{\Psi}_{2}\left(\operatorname{Sp}_{2 n}\right)$ is written as

$$
\psi=(\tau, 2 b+1) \boxplus(\tau, 1) \boxplus \bigoplus_{i=3}^{r}\left(\tau_{i}, 1\right),
$$

where $b \geq 1$ and $\tau \not \tau_{i}$ for any $3 \leq i \leq r$. Assume that $\tau \in \mathcal{A}_{\text {cusp }}\left(\mathrm{GL}_{a}\right)$ has central character $\omega_{\tau}$, and $\tau_{i} \in \mathcal{A}_{\text {cusp }}\left(\mathrm{GL}_{a_{i}}\right)$ has central character $\omega_{\tau_{i}}$ for $3 \leq i \leq r$. Then $2 n+1=a(2 b+1)+a+\sum_{i=3}^{r} a_{i}$ and $\omega_{\tau}^{2 b+1} \cdot \omega_{\tau} \cdot \prod_{i=3}^{r} \omega_{\tau_{i}}=1$. Consider the isobaric representation $\pi=\tau_{3} \boxplus \cdots \boxplus \tau_{r}$ of $\mathrm{GL}_{2 m+1}(\mathbb{A})$, where $2 m+1=$ $\sum_{i=3}^{r} a_{i}=2 n+1-a(2 b+2)$. Then $\pi$ has central character $\omega_{\pi}=\prod_{i=3}^{r} \omega_{\tau_{i}}=1$.

By [Ginzburg et al. 2011, Theorem 3.1], there is a generic $\sigma \in \mathcal{A}_{\text {cusp }}\left(\operatorname{Sp}_{2 m}\right)$ such that $\sigma$ has the functorial transfer $\pi$ and hence $L(s, \tau \times \sigma)$ is holomorphic at $s=1$ in this case. For any automorphic form

$$
\phi \in \mathcal{A}\left(N_{a(b+1)}(\mathbb{A}) M_{a(b+1)}(F) \backslash \operatorname{Sp}_{2 a(b+1)+2 m}(\mathbb{A})\right)_{\Delta(\tau, b+1) \otimes \sigma},
$$

one defines a residual Eisenstein series as in Case I

$$
E(\phi, s)(g)=E\left(g, \phi_{\Delta(\tau, b+1) \otimes \sigma}, s\right) .
$$

By [Jiang et al. 2013], this Eisenstein series has a simple pole at $b / 2$, which is the right-most one. Denote the representation generated by these residues at $s=b / 2$ by $\mathcal{E}_{\Delta(\tau, b+1) \otimes \sigma}$, which is square-integrable. Following [Jiang et al. 2013] and [Shahidi 2010, Theorem 7.1.2], this residual representation $\mathcal{E}_{\Delta(\tau, b+1) \otimes \sigma}$ is nonzero. In particular, by Section 6.2 of [Jiang et al. 2013], the global Arthur parameter of $\mathcal{E}_{\Delta(\tau, b+1) \otimes \sigma}$ is exactly $\psi=(\tau, 2 b+1) \boxplus(\tau, 1) \boxplus \boxplus_{i=3}^{r}\left(\tau_{i}, 1\right)$ as in Case II. In this case, we prove

Theorem 2.5. For any global Arthur parameter of the form

$$
\psi=(\tau, 2 b+1) \boxplus(\tau, 1) \boxplus \bigoplus_{i=3}^{r}\left(\tau_{i}, 1\right)
$$

with $b \geq 1$ and $\tau \neq \tau_{i}$ for any $3 \leq i \leq r$, the residual representation $\mathcal{E}_{\Delta(\tau, b+1) \otimes \sigma}$ has a nonzero Fourier coefficient attached to the Barbasch-Vogan duality

$$
\eta_{\mathfrak{s o}_{2 n+1}, \mathfrak{s p}_{2 n}}(\underline{p}(\psi))
$$

of the partition $\underline{p}(\psi)$ associated to $\left(\psi, \mathrm{SO}_{2 n+1}(\mathbb{C})\right)$.

Following [Jiang 2014, Section 4], $p(\psi)=\left[(2 b+1)^{a}(1)^{a}(1)^{2 m+1}\right]$. Now by Definition 2.2, we may calculate the partition $\eta_{\mathfrak{s o}_{2 n+1}, \mathfrak{s p}_{2 n}}(\underline{p}(\psi))$ explicitly as 
follows. When $a$ is even,

$$
\begin{aligned}
\eta_{\mathfrak{s o}_{2 n+1}, \mathfrak{s p}_{2 n}}(\underline{p}(\psi)) & =\eta_{\mathfrak{s o}_{2 n+1}, \mathfrak{s p}_{2 n}}\left(\left[(2 b+1)^{a}(1)^{2 m+1+a}\right]\right) \\
& =\left[(2 b+1)^{a}(1)^{2 m+a}\right]^{t} \\
& =\left[(a)^{2 b+1}\right]+[(2 m+a)] \\
& =\left[(2 m+2 a)(a)^{2 b}\right] .
\end{aligned}
$$

When $a$ is odd,

$$
\begin{aligned}
\eta_{\mathfrak{s o}_{2 n+1}, \mathfrak{s p}_{2 n}}(\underline{p}(\psi)) & =\eta_{\mathfrak{s o}_{2 n+1}, \mathfrak{s p}_{2 n}}\left(\left[(2 b+1)^{a}(1)^{2 m+1+a}\right]\right) \\
& =\left(\left[(2 b+1)^{a}(1)^{2 m+a}\right]_{\mathrm{Sp}_{2 n}}\right)^{t} \\
& =\left[(2 b+1)^{a-1}(2 b)(2)(1)^{2 m+a-1}\right]^{t} \\
& =\left[(a-1)^{2 b+1}\right]+\left[(1)^{2 b}\right]+\left[(1)^{2}\right]+[(2 m+a-1)] \\
& =\left[(2 m+2 a)(a+1)(a)^{2 b-2}(a-1)\right] .
\end{aligned}
$$

As before, if $a$ is odd, then, by the recipe for obtaining the $\mathrm{Sp}_{2 n}$-expansion of a symplectic partition $\underline{p}$ given in [Collingwood and McGovern 1993, Lemma 6.3.9],

$$
\left[(2 m+2 a)(a+1)(a)^{2 b-2}(a-1)\right]=\left[(2 m+2 a)(a)^{2 b}\right]^{\mathrm{Sp}_{2 n}} .
$$

Hence it suffices to prove the following theorem.

Theorem 2.6. The residual representation $\mathcal{E}_{\Delta(\tau, b+1) \otimes \sigma}$ has a nonzero Fourier coefficient attached to $\left[(2 m+2 a)(a)^{2 b}\right]$.

The proof of Theorem 2.6 is given in Section 6, using induction on the integer $b$. We note that when $b=0$, the Arthur parameter is

$$
\psi=2(\tau, 1) \boxplus \bigoplus_{i=3}^{r}\left(\tau_{i}, 1\right),
$$

which does not parametrize automorphic representations in the discrete spectrum. Indeed, in this case, the corresponding automorphic representation constructed from the Eisenstein series is the value at $s=0$, which we still denote by $\mathcal{E}_{\Delta(\tau, 1) \otimes \sigma}=\mathcal{E}_{\tau \otimes \sigma}$. It is clear that in this case, the partition $p(\psi)$ is the trivial partition. On the other hand, following [Shahidi 2010, Theorem 7.1.3], the representation $\mathcal{E}_{\Delta(\tau, 1) \otimes \sigma}$ has a nonzero Whittaker-Fourier coefficient. In other words, Theorem 2.6 still holds for $b=0$. As we proceed in Section 6 , the case of $b=0$ will serve as the base of the induction argument.

2C. Case III. $\psi \in \widetilde{\Psi}_{2}\left(\mathrm{Sp}_{2 n}\right)$ is written as

$$
\psi=(\tau, 2 b) \boxplus \bigoplus_{i=2}^{r}\left(\tau_{i}, 1\right),
$$


where $b \geq 1$. In this case, $\tau$ is of symplectic type (and hence $a=2 k$ is even), while $\tau_{i}$ for all $2 \leq i \leq r$ are of orthogonal type. Assume that $\tau \in \mathcal{A}_{\text {cusp }}\left(\mathrm{GL}_{a}\right)$ has central character $\omega_{\tau}$, and $\tau_{i} \in \mathcal{A}_{\text {cusp }}\left(\mathrm{GL}_{a_{i}}\right)$ has central character $\omega_{\tau_{i}}$ for $2 \leq i \leq r$. By the definition of Arthur parameters, one has that $2 n+1=2 a b+\sum_{i=2}^{r} a_{i}$, and $\prod_{i=2}^{r} \omega_{\tau_{i}}=1$. Consider the isobaric representation $\pi=\tau_{2} \boxplus \cdots \boxplus \tau_{r}$ of $\mathrm{GL}_{2 m+1}(\mathbb{A})$, where $2 m+1=\sum_{i=2}^{r} a_{i}=2 n+1-2 a b$. Hence $\pi$ has central character $\omega_{\pi}=\prod_{i=2}^{r} \omega_{\tau_{i}}=1$.

By [Ginzburg et al. 2011, Theorem 3.1], there is a generic $\sigma \in \mathcal{A}_{\text {cusp }}\left(\operatorname{Sp}_{2 m}\right)$ that has the functorial transfer $\pi$. Then we define a residual Eisenstein series

$$
E(\phi, s)(g)=E\left(g, \phi_{\Delta(\tau, b) \otimes \sigma}, s\right)
$$

associated to any automorphic form

$$
\phi \in \mathcal{A}\left(N_{a b}(\mathbb{A}) M_{a b}(F) \backslash \operatorname{Sp}_{2 a b+2 m}(\mathbb{A})\right)_{\Delta(\tau, b) \otimes \sigma} .
$$

By [Jiang et al. 2013], this Eisenstein series may have a simple pole at $b / 2$, which is the right-most one. Denote the representation generated by these residues at $s=b / 2$ by $\mathcal{E}_{\Delta(\tau, b) \otimes \sigma}$. This residual representation is square-integrable. If $L\left(\frac{1}{2}, \tau \times \sigma\right) \neq 0$, the residual representation $\mathcal{E}_{\tau \otimes \sigma}$ is nonzero, and hence by the induction argument in [Jiang et al. 2013], the residual representation $\mathcal{E}_{\Delta(\tau, b) \otimes \sigma}$ is also nonzero. Finally, following [op. cit., Section 6.2], we see that the global Arthur parameter of $\mathcal{E}_{\Delta(\tau, b) \otimes \sigma}$ is exactly $\psi=(\tau, 2 b) \boxplus \boxplus_{i=2}^{r}\left(\tau_{i}, 1\right)$ as in (2-3).

Theorem 2.7. Assume that $a=2 k$ and $L\left(\frac{1}{2}, \tau \times \sigma\right) \neq 0$. If the residual representation $\mathcal{E}_{\tau \otimes \sigma}$ of $\operatorname{Sp}_{4 k+2 m}(\mathbb{A})$, with $\sigma \not 1_{\mathrm{Sp}_{0}(\mathbb{A})}$, has a nonzero Fourier coefficient attached to the partition $[(2 k+2 m)(2 k)]$, then, for any $b \geq 1$, the residual representation $\mathcal{E}_{\Delta(\tau, b) \otimes \sigma}$ has a nonzero Fourier coefficient attached to the partition $\left[(2 k+2 m)(2 k)^{2 b-1}\right]$.

We remark that if $\sigma \cong 1_{\operatorname{Sp}_{0}(\mathbb{A})},\left(\frac{1}{2},\right)=L\left(\frac{1}{2}, \tau \times \sigma\right) \neq 0$. In this case, [Liu 2013a, Theorem 4.2.2] shows that $\mathfrak{p}^{m}\left(\mathcal{E}_{\Delta(\tau, b) \otimes \sigma}\right)=\left\{\left[(2 k)^{2 b}\right]\right\}$.

In fact, the assumption that the residual representation $\mathcal{E}_{\tau \otimes \sigma}$ of $\operatorname{Sp}_{4 k+2 m}(\mathbb{A})$, with $\sigma \not 1_{\mathrm{Sp}_{0}(\mathbb{A})}$, has a nonzero Fourier coefficient attached to the partition $[(2 k+2 m)(2 k)]$ is exactly [Ginzburg et al. 2004, Conjecture 6.1], and hence Theorem 2.7 has a close connection to the Gan-Gross-Prasad conjecture [Gan et al. 2012]. We will come back to this issue in our future work.

In this case, $\underline{p}(\psi)=\left[(2 b)^{a}(1)^{2 m+1}\right]$, and following the calculation in [Jiang and Liu 2015b],

$$
\eta_{\mathfrak{s o}_{2 n+1}, \mathfrak{s p}_{2 n}}(p(\psi))=\left[(a+2 m)(a)^{2 b-1}\right],
$$

where $a=2 k$ is even. The proof of Theorem 2.7 is given in Section 7 . 
When $L\left(\frac{1}{2}, \tau \times \sigma\right)$ is zero for the Arthur parameter in (2-3), the corresponding automorphic $L^{2}$-packet $\widetilde{\Pi}_{\psi}\left(\epsilon_{\psi}\right)$ are expected to contain all cuspidal automorphic representations if it is not empty. We are going to apply the construction of endoscopy correspondences outlined in [Jiang 2014] to construct the distinguished cuspidal members in $\widetilde{\Pi}_{\psi}\left(\epsilon_{\psi}\right)$. The details for this case will be considered in our future work. See [Jiang and Liu 2015b] for a brief discussion in this aspect.

\section{A basic lemma}

We recall a basic lemma from [Jiang and Liu 2015b], which will be a technical key step in the proofs of this paper. Let $H$ be a reductive group defined over $F$. We first recall [Jiang and Liu 2013, Lemma 5.2], which is also formulated in a slightly different version in [Ginzburg et al. 2011, Corollary 7.1]. Note that the proof of [Jiang and Liu 2013, Lemma 5.2] is valid for $H(\mathbb{A})$.

Let $C$ be an $F$-subgroup of a maximal unipotent subgroup of $H$, and let $\psi_{C}$ be a nontrivial character of $[C]=C(F) \backslash C(\mathbb{A})$. Suppose that $\tilde{X}, \tilde{Y}$ are two unipotent $F$-subgroups, satisfying the following conditions:

(1) $\tilde{X}$ and $\tilde{Y}$ normalize $C$;

(2) $\tilde{X} \cap C$ and $\tilde{Y} \cap C$ are normal in $\tilde{X}$ and $\tilde{Y}$, respectively, $(\tilde{X} \cap C) \backslash \tilde{X}$ and $(\tilde{Y} \cap C) \backslash \tilde{Y}$ are abelian;

(3) $\tilde{X}(\mathbb{A})$ and $\tilde{Y}(\mathbb{A})$ preserve $\psi_{C}$;

(4) $\psi_{C}$ is trivial on $(\tilde{X} \cap C)(\mathbb{A})$ and $(\tilde{Y} \cap C)(\mathbb{A})$;

(5) $[\tilde{X}, \tilde{Y}] \subset C$;

(6) there is a nondegenerate pairing $(\tilde{X} \cap C)(\mathbb{A}) \times(\tilde{Y} \cap C)(\mathbb{A}) \rightarrow \mathbb{C}^{*}$, given by $(x, y) \mapsto \psi_{C}([x, y])$, which is multiplicative in each coordinate, and identifies $(\tilde{Y} \cap C)(F) \backslash \tilde{Y}(F)$ and $(\tilde{X} \cap C)(F) \backslash \tilde{X}(F)$ with the duals of the subgroups $\tilde{X}(F)(\tilde{X} \cap C)(\mathbb{A}) \backslash \tilde{X}(\mathbb{A})$ and $\tilde{Y}(F)(\tilde{Y} \cap C)(\mathbb{A}) \backslash \tilde{Y}(\mathbb{A})$, respectively.

Let $B=C \tilde{Y}$ and $D=C \tilde{X}$, and extend $\psi_{C}$ trivially to characters of $[B]=$ $B(F) \backslash B(\mathbb{A})$ and $[D]=D(F) \backslash D(\mathbb{A})$, which will be denoted by $\psi_{B}$ and $\psi_{D}$, respectively.

Lemma 3.1 [Jiang and Liu 2013, Lemma 5.2]. Assume that $\left(C, \psi_{C}, \tilde{X}, \tilde{Y}\right)$ satisfies all the above conditions. Let $f$ be an automorphic form on $H(\mathbb{A})$. Then

$$
\int_{[C]} f(c g) \psi_{C}^{-1}(c) d c \equiv 0, \quad \text { for all } g \in H(\mathbb{A}),
$$

if and only if

$$
\int_{[D]} f(u g) \psi_{D}^{-1}(u) d u \equiv 0, \quad \text { for all } g \in H(\mathbb{A})
$$


if and only if

$$
\int_{[B]} f(v g) \psi_{B}^{-1}(v) d v \equiv 0, \quad \text { for all } g \in H(\mathbb{A}) .
$$

For simplicity, we always use $\psi_{C}$ to denote its extensions $\psi_{B}$ and $\psi_{D}$ when we apply Lemma 3.1 to various circumstances. Lemma 3.1 can be extended as follows and will be a technical key in this paper.

Lemma 3.2 [Jiang and Liu 2015b, Lemma 6.2]. Assume that $\left(C, \psi_{C}, \tilde{X}, \tilde{Y}\right)$ satisfies the following conditions: $\tilde{X}=\left\{\tilde{X}_{i}\right\}_{i=1}^{r}, \tilde{Y}=\left\{\tilde{Y}_{i}\right\}_{i=1}^{r}$, and for $1 \leq i \leq r$, each quadruple

$$
\left(\tilde{X}_{i-1} \cdots \tilde{X}_{1} C \tilde{Y}_{r} \cdots \tilde{Y}_{i+1}, \psi_{C}, \tilde{X}_{i}, \tilde{Y}_{i}\right)
$$

satisfies all the conditions of Lemma 3.1. Let $f$ be an automorphic form on $H(\mathbb{A})$. Then

$$
\int_{\left[\tilde{X}_{r} \cdots \tilde{X}_{1} C\right]} f(x c g) \psi_{C}^{-1}(c) d c d x \equiv 0, \quad \text { for all } g \in H(\mathbb{A}),
$$

if and only if

$$
\int_{\left[C \tilde{Y}_{r} \cdots \tilde{Y}_{1}\right]} f(c y g) \psi_{C}^{-1}(c) d y d c \equiv 0, \quad \text { for all } g \in H(\mathbb{A}) .
$$

The proof of this lemma is carried out by using Lemma 3.1 inductively, and was given with full details in [loc. cit.].

\section{Proof of part (1) of Theorem 2.4}

In this section, we assume that $a=2 m+1$ and show that $\mathcal{E}_{\Delta(\tau, b) \otimes \sigma}$ has a nonzero Fourier coefficient attached to $\underline{p}:=\left[(2 m+1)^{2 b}(2 m)\right]$.

Proof of part (1) of Theorem 2.4. We will prove the theorem by induction on $b$. Note that when $b=0, \mathcal{E}_{\Delta(\tau, b) \otimes \sigma} \cong \sigma$ which has a nonzero Fourier coefficient attached to $[(2 m)]$ since $\sigma$ is generic. Now assume that $\mathcal{E}_{\Delta(\tau, b-1) \otimes \sigma}$ has a nonzero $\psi_{\left[(2 m+1)^{2 b-2}(2 m)\right], \alpha}$-Fourier coefficient attached to $\left[(2 m+1)^{2 b-2}(2 m)\right]$, for some $\alpha \in F^{*} /\left(F^{*}\right)^{2}$.

Take any $\varphi \in \mathcal{E}_{\Delta(\tau, b) \otimes \sigma}$ and consider its $\psi_{\underline{p}, \alpha}$-Fourier coefficients attached to $\underline{p}$ :

$$
\varphi^{\psi_{\underline{p}, \alpha}}(g)=\int_{\left[V_{\underline{p}, 2}\right]} \varphi(v g) \psi_{\underline{p}, \alpha}^{-1}(v) d v .
$$

For definitions of the unipotent group $V_{\underline{p}, 2}$ and its character $\psi_{\underline{p}, \alpha}$, see [Jiang and Liu 2015c, Section 2]. By [op. cit., Corollary 2.4], the integral in (4-1) is nonvanishing if and only if the following integral is nonvanishing:

$$
\int_{\left[Y_{1} V_{\underline{p}, 2}\right]} \varphi(v g) \psi_{\underline{p}, \alpha}^{-1}(v) d v,
$$


where $Y_{1}$ is defined in [Jiang and Liu 2015c, (2.5)] corresponding to the partition $\left[(2 m+1)^{2 b}(2 m)\right]$ and the character $\psi_{p, \alpha}$ extends to $Y_{1} V_{p, 2}$ trivially.

Assume that $T$ is the maximal split torus in $\operatorname{Sp}_{2 b(2 m+1)+2 m}$, consisting of elements

$$
\operatorname{diag}\left(t_{1}, t_{2}, \ldots, t_{b(2 m+1)+m}, t_{b(2 m+1)+m}^{-1}, \ldots, t_{2}^{-1}, t_{1}^{-1}\right) .
$$

Let $\omega_{1}$ be the Weyl element of $\operatorname{Sp}_{2 b(2 m+1)+2 m}$, sending elements $t \in T$ to the torus elements

$$
t^{\prime}=\operatorname{diag}\left(t^{(0)}, t^{(1)}, t^{(2)}, \ldots, t^{(m)}, t^{(m+1)}, t^{(m), *}, \ldots, t^{(2), *}, t^{(1), *}, t^{(0), *}\right),
$$

where $t^{(0)}=\operatorname{diag}\left(t_{1}, t_{2}, \ldots, t_{2 m+1}\right)$, and with $e=2 m+1$,

$$
t^{(m+1)}=\operatorname{diag}\left(t_{e+m+1}, \ldots, t_{(b-1) e+m+1}, t_{b e-m}^{-1}, \ldots, t_{2 e-m}^{-1}\right)
$$

and

$$
t^{(j)}=\operatorname{diag}\left(t_{e+j}, \ldots, t_{(b-1) e+j}, t_{b e-j+1}^{-1}, \ldots, t_{2 e-j+1}^{-1}, t_{b e+j}\right),
$$

for $1 \leq j \leq m$.

Now identify $\mathrm{Sp}_{(2 b-1)(2 m+1)+2 m}$ with its image in $\mathrm{Sp}_{2 b(2 m+1)+2 m}$ under the embedding $g \mapsto \operatorname{diag}\left(I_{2 m+1}, g, I_{2 m+1}\right)$, and denote the restriction of $\omega_{1}$ to $\operatorname{Sp}_{(2 b-1)(2 m+1)+2 m}$ by $\omega_{1}^{\prime}$. We conjugate cross the integration variables by $\omega_{1}$ from the left; then the integral in (4-2) becomes

$$
\int_{\left[U_{\underline{p}, 2}\right]} \varphi\left(u \omega_{1} g\right) \psi_{\underline{p}, \alpha}^{\omega_{1}}(u)^{-1} d u,
$$

where $U_{\underline{p}, 2}=\omega_{1} Y_{1} V_{\underline{p}, 2} \omega_{1}^{-1}$, and $\psi_{\underline{p}, \alpha}^{\omega_{1}}(u)=\psi_{\underline{p}, \alpha}\left(\omega_{1}^{-1} u \omega_{1}\right)$.

Now, we describe the structure of elements in $U_{\underline{p}, 2}$, each of which has the form

$$
u=\left(\begin{array}{ccc}
z_{2 m+1} & q_{1} & q_{2} \\
0 & u^{\prime} & q_{1}^{*} \\
0 & 0 & z_{2 m+1}^{*}
\end{array}\right)\left(\begin{array}{ccc}
I_{2 m+1} & 0 & 0 \\
p_{1} & I_{(2 b-2)(2 m+1)+2 m} & 0 \\
p_{2} & p_{1}^{*} & I_{2 m+1}
\end{array}\right),
$$

where $z_{2 m+1} \in V_{2 m+1}$, the standard maximal unipotent subgroup of $\mathrm{GL}_{2 m+1}$; $u^{\prime} \in U_{\left[(2 m+1)^{2 b-2}(2 m)\right], 2}:=\omega_{1}^{\prime} Y_{2} V_{\left[(2 m+1)^{2 b-2}(2 m)\right], 2} \omega_{1}^{\prime-1}$ with $Y_{2}$ as in [Jiang and Liu 2015c, (2.5)] corresponding to the partition $\left[(2 m+1)^{2 b-2}(2 m)\right]$; and $p_{i}, q_{i}, 1 \leq i \leq 2$, are described as follows:

- $q_{1} \in M_{(2 m+1) \times((2 b-2)(2 m+1)+2 m)}$, such that $q_{1}(i, j)=0$ for $1 \leq i \leq 2 m+1$ and $1 \leq j \leq(2 b-2)+(2 b-1)(i-1)$.

- $p_{1} \in M_{((2 b-2)(2 m+1)+2 m) \times(2 m+1)}$, such that $p_{1}(i, j)=0$ for $1 \leq j \leq 2 m+1$ and $(2 b-2)+(2 b-1)(i-1)+1 \leq i \leq(2 b-2)(2 m+1)+2 m$.

- $q_{2} \in M_{(2 m+1) \times(2 m+1)}$, symmetric with respect to the secondary diagonal, such that $q_{2}(i, j)=0$ for $1 \leq i \leq 2 m+1$ and $1 \leq j \leq i$. 
- $p_{2} \in M_{(2 m+1) \times(2 m+1)}$, symmetric with respect to the secondary diagonal, such that $p_{2}(i, j)=0$ for $1 \leq i \leq 2 m+1$ and $1 \leq j \leq i$.

Note that

$$
\psi_{\underline{p}, \alpha}^{\omega_{1}}\left(\begin{array}{ccc}
z_{2 m+1} & q_{1} & q_{2} \\
0 & I_{(2 b-2)(2 m+1)+2 m} & q_{1}^{*} \\
0 & 0 & z_{2 m+1}^{*}
\end{array}\right)=\psi\left(\sum_{i=1}^{2 m} z_{2 m+1}(i, i+1)\right) .
$$

Next, we apply Lemma 3.2 to fill the zero entries in $q_{1}, q_{2}$ using the nonzero entries in $p_{1}, p_{2}$. To proceed, we need to define a sequence of one-dimensional root subgroups and put them in a correct order.

Let $X_{j}$, with $1 \leq j \leq(2 b-2)+1$, be the one-dimensional subgroups corresponding to the roots such that the corresponding entries are in the first row of $q_{1}$ or $q_{2}$ and are identically zero, from right to left. For $1<i \leq m$, let $X_{j}$, with

$$
\left(\sum_{k=1}^{i-1}[(2 b-2)+(2 b-1)(k-1)+k]\right)+1 \leq j \leq \sum_{k=1}^{i}[(2 b-2)+(2 b-1)(k-1)+k],
$$

be the one-dimensional subgroups corresponding to the roots such that the corresponding entries are in the $i$-th row of $q_{1}$ or $q_{2}$ and are identically zero, from right to left.

Let $Y_{j}$, with $1 \leq j \leq(2 b-2)+1$, be the one-dimensional subgroups corresponding to the roots such that the corresponding entries are in the second column of $p_{1}$ or $p_{2}$ and are not identically zero, from bottom to top. For $1<i \leq m$, let $Y_{j}$, with

$$
1+\sum_{k=1}^{i-1}[(2 b-2)+(2 b-1)(k-1)+k] \leq j \leq \sum_{k=1}^{i}[(2 b-2)+(2 b-1)(k-1)+k],
$$

be the one-dimensional subgroups corresponding to the roots such that the corresponding entries are in the $(i+1)$-th column of $p_{1}$ or $p_{2}$ and are not identically zero, from bottom to top.

Let $W_{1}$ be the subgroup of $U_{p, 2}$ such that the entries corresponding to the one-dimensional subgroups $Y_{j}$ above, with

$$
1 \leq j \leq \ell:=\sum_{k=1}^{m}[(2 b-2)+(2 b-1)(k-1)+k],
$$

are all identically zero. And let $\psi_{W_{1}}=\left.\psi_{\underline{p}, \alpha}^{\omega_{1}}\right|_{W_{1}}$. Then $\left(W_{1}, \psi_{W_{1}},\left\{X_{j}\right\}_{j}^{\ell},\left\{Y_{j}\right\}_{j}^{\ell}\right)$ satisfies all the conditions for Lemma 3.2. Hence, by that lemma, the integral in (4-4) is nonvanishing if and only if the following integral is nonvanishing:

$$
\int_{\left[W_{2}\right]} \varphi\left(w \omega_{1} g\right) \psi_{W_{2}}(w)^{-1} d w,
$$


where $W_{2}:=\prod_{j=1}^{\ell} X_{j} W_{1}$ and $\psi_{W_{2}}$ is the character on $W_{2}$ extended trivially from $\psi_{W_{1}}$.

Now we consider the $i$-th row of $q_{1}$ and $q_{2}$, with $m+1 \leq i \leq 2 m$. We will continue to apply Lemma 3.2 to fill the zero entries in $q_{1}$ and $q_{2}$, row by row, from the $(m+1)$-th row to $2 m$-th row. But for each $m+1 \leq i \leq 2 m$, before we apply Lemma 3.2 as above, we need to take the Fourier expansion along the one-dimensional root subgroup $X_{2 e_{i}}$. For example, for $i=m+1$, we first take the Fourier expansion of the integral in (4-6) along the one-dimensional root subgroup $X_{2 e_{m+1}}$. We will get two kinds of Fourier coefficients corresponding to the orbits of the dual of $\left[X_{2 e_{m+1}}\right]:=X_{2 e_{m+1}}(F) \backslash X_{2 e_{m+1}}(\mathbb{A})$ : the trivial orbit and the nontrivial one. For the Fourier coefficients attached to the nontrivial orbit, we can see that there is an inner integral

$$
\varphi^{\psi_{[(2 m+2) 12 b(2 m+1)-2], \beta}, \quad \beta \in F^{*},}
$$

which is identically zero by [Jiang and Liu 2015a, Proposition 6.4]. Therefore only the Fourier coefficient attached to the trivial orbit, which actually equals to the integral in (4-6), survives. Then, we can apply the Lemma 3.2 to the $(m+1)$-th row of $q_{1}$ and $q_{2}$ similarly as above.

After considering all the $i$-th row of $q_{1}$ and $q_{2}, m+1 \leq i \leq 2 m$ as above, we get that the integral in (4-6) is nonvanishing if and only if the following integral is nonvanishing:

$$
\int_{\left[W_{3}\right]} \varphi\left(w \omega_{1} g\right) \psi_{W_{3}}(w)^{-1} d w,
$$

where $W_{3}$ has elements of the following form:

$$
w=\left(\begin{array}{ccc}
z_{2 m+1} & q_{1} & q_{2} \\
0 & u^{\prime} & q_{1}^{*} \\
0 & 0 & z_{2 m+1}^{*}
\end{array}\right),
$$

where $z_{2 m+1} \in V_{2 m+1}$, the standard maximal unipotent subgroup of $\mathrm{GL}_{2 m+1}$;

$$
u^{\prime} \in U_{\left[(2 m+1)^{2 b-2}(2 m)\right], 2}:=\omega_{1}^{\prime} Y_{2} V_{\left[(2 m+1)^{2 b-2}(2 m)\right], 2} \omega_{1}^{-1}
$$

with $Y_{2}$ as in [op. cit., (2.5)] corresponding to the partition $\left[(2 m+1)^{2 b-2}(2 m)\right]$;

$$
q_{1} \in M_{(2 m+1) \times((2 b-2)(2 m+1)+2 m)},
$$

such that $q_{1}(2 m+1, j)=0$ for $1 \leq j \leq(2 b-2)(2 m+1)+2 m$;

$$
q_{2} \in M_{(2 m+1) \times(2 m+1)},
$$


symmetric with respect to the secondary diagonal, such that $q_{2}(2 m+1,1)=0$. Also,

$$
\psi_{W_{3}}\left(\begin{array}{ccc}
z_{2 m+1} & q_{1} & q_{2} \\
0 & I_{(2 b-2)(2 m+1)+2 m} & q_{1}^{*} \\
0 & 0 & z_{2 m+1}^{*}
\end{array}\right)=\psi\left(\sum_{i=1}^{2 m} z_{2 m+1}(i, i+1)\right) .
$$

Now consider the Fourier expansion of the integral in (4-7) along the onedimensional root subgroup $X_{2 e_{2 m+1}}$. By the same reason as above, only the Fourier coefficient corresponding to the trivial orbit of the dual of $\left[X_{2 e_{2 m+1}}\right]$ survives, which is actually equal to the integral in (4-7):

$$
\int_{\left[W_{4}\right]} \varphi\left(w \omega_{1} g\right) \psi_{W_{4}}(w)^{-1} d w,
$$

where elements in $W_{4}$ have the same structure as in (4-8), except that $q_{2}(2 m+1,1)$ is not identically zero.

It is easy to see that the integral in (4-9) has an inner integral which is exactly $\varphi^{\psi_{N_{12 m}}}$, using notation in Lemma 4.2 below. On the other hand, we know that by Lemma 4.2 below, $\varphi^{\psi_{N_{12 m}}}=\varphi^{\widetilde{\psi}_{N_{12 m+1}}}$. Therefore, the integral in (4-9) becomes

$$
\int_{\left[W_{5}\right]} \varphi\left(w \omega_{1} g\right) \psi_{W_{5}}(w)^{-1} d w,
$$

where elements in $W_{5}$ are of the form:

$$
w=w\left(z_{2 m+1}, u^{\prime}, q_{1}, q_{2}\right)=\left(\begin{array}{ccc}
z_{2 m+1} & q_{1} & q_{2} \\
0 & u^{\prime} & q_{1}^{*} \\
0 & 0 & z_{2 m+1}^{*}
\end{array}\right),
$$

where $z_{2 m+1} \in V_{2 m+1}$, the standard maximal unipotent subgroup of $\mathrm{GL}_{2 m+1}$;

$$
u^{\prime} \in U_{\left[(2 m+1)^{2 b-2}(2 m)\right], 2}:=\omega_{1}^{\prime} Y_{2} V_{\left[(2 m+1)^{2 b-2}(2 m)\right], 2} \omega_{1}^{\prime-1}
$$

with $Y_{2}$ as in [loc. cit.] corresponding to the partition $\left[(2 m+1)^{2 b-2}(2 m)\right]$;

$$
q_{1} \in M_{(2 m+1) \times((2 b-2)(2 m+1)+2 m)},
$$

and $q_{2} \in M_{(2 m+1) \times(2 m+1)}$, symmetric with respect to the secondary diagonal. And

$$
\psi_{W_{5}}\left(\begin{array}{ccc}
z_{2 m+1} & q_{1} & q_{2} \\
0 & I_{(2 b-2)(2 m+1)+2 m} & q_{1}^{*} \\
0 & 0 & z_{2 m+1}^{*}
\end{array}\right)=\psi\left(\sum_{i=1}^{2 m} z_{2 m+1}(i, i+1)\right) .
$$

Hence, the integral in (4-10) can be written as

$$
\int_{W_{6}} \varphi_{P_{2 m+1}}\left(w \omega_{1} g\right) \psi_{W_{6}}(w)^{-1} d w,
$$

where $W_{6}$ is a subgroup of $W_{5}$ consisting of elements of the form $w\left(z_{2 m+1}, u^{\prime}, 0,0\right)$, $\psi_{W_{6}}=\left.\psi_{W_{5}}\right|_{W_{6}}$, and $\varphi_{P_{2 m+1}}$ is the constant term of $\varphi$ along the parabolic subgroup 
$P_{2 m+1}=M_{2 m+1} N_{2 m+1}$ of $\mathrm{Sp}_{2 b(2 m+1)+2 m}$ with the Levi subgroup isomorphic to $\mathrm{GL}_{2 m+1} \times \mathrm{Sp}_{(2 b-2)(2 m+1)+2 m}$.

By Lemma 4.1 below, $\varphi_{P_{2 m+1}}\left(w \omega_{1} g\right)$ is an automorphic form in $\tau|\cdot|^{-b} \otimes$ $\mathcal{E}_{\Delta(\tau, b-1) \otimes \sigma}$ when restricted to the Levi subgroup. Note that the restriction of $\psi_{W_{5}}$ to the $z_{2 m+1}$-part gives a Whittaker coefficient of $\tau$, and the restriction to the $u^{\prime}$-part gives a $\psi_{\left[(2 m+1)^{2 b-2}(2 m)\right], \alpha}$-Fourier coefficient of $\mathcal{E}_{\Delta(\tau, b-1) \otimes \sigma}$ up to the conjugation of the Weyl element $\omega_{1}^{\prime}$. On the other hand, $\tau$ is generic, and by induction assumption, $\mathcal{E}_{\Delta(\tau, b-1) \otimes \sigma}$ has a nonzero $\psi_{\left[(2 m+1)^{2 b-2}(2 m)\right], \alpha}$-Fourier coefficient. Therefore, we conclude that $\mathcal{E}_{\Delta(\tau, b) \otimes \sigma}$ has a nonzero $\psi_{p, \alpha}$-Fourier coefficient attached to the partition $p=\left[(2 m+1)^{2 b}(2 m)\right]$. This completes the proof of part (1) of Theorem 2.4, up to Lemmas 4.1 and 4.2, which are stated below.

Note that Lemmas 4.1 and 4.2 are analogs of [Liu 2013a, Lemmas 4.2.4 and 4.2.6], with similar arguments, and hence we state them without proofs.

Lemma 4.1. Let $P_{a i}=M_{a i} N_{a i}$, with $1 \leq i \leq b$ and $a \leq 2 m+1$, be the parabolic subgroup of $\mathrm{Sp}_{2 a b+2 m}$ with Levi part

$$
M_{a i} \cong \mathrm{GL}_{a i} \times \mathrm{Sp}_{a(2 b-2 i)+2 m} .
$$

Let $\varphi$ be an arbitrary automorphic form in $\mathcal{E}_{\Delta(\tau, b) \otimes \sigma}$. Denote by $\varphi_{P_{a i}}(g)$ the constant term of $\varphi$ along $P_{a i}$. Then, for $1 \leq i \leq b$,

$$
\varphi_{P_{a i}} \in \mathcal{A}\left(N_{a i}(\mathbb{A}) M_{a i}(F) \backslash \operatorname{Sp}_{2 a b+2 m}(\mathbb{A})\right)_{\Delta(\tau, i)|\cdot|^{-(2 b+1-i) / 2} \otimes \mathcal{E}_{\Delta(\tau, b-i) \otimes \sigma}}
$$

Note that when $b=i, \mathcal{E}_{\Delta(\tau, b-i) \otimes \sigma}=\sigma$.

Lemma 4.2. Let $N_{1^{p}}$ be the unipotent radical of the parabolic subgroup $P_{1^{p}}$ of $\mathrm{Sp}_{2 b(2 m+1)+2 m}$ with the Levi part being $\mathrm{GL}_{1}^{\times p} \times \mathrm{Sp}_{2 b(2 m+1)+2 m-2 p}$. Let

$$
\psi_{N_{1} p}(n):=\psi\left(n_{1,2}+\cdots+n_{p, p+1}\right) \quad \text { and } \quad \tilde{\psi}_{N_{1^{p}}}(n):=\psi\left(n_{1,2}+\cdots+n_{p-1, p}\right)
$$

be two characters of $N_{1^{p}}$. For any automorphic form $\varphi \in \mathcal{E}_{\Delta(\tau, b) \otimes \sigma}$, define $\psi_{N_{1^{p}}}$ and $\tilde{\psi}_{N_{1} p}$-Fourier coefficients as follows:

$$
\varphi^{\psi_{N_{1} p}}(g):=\int_{\left[N_{1} p\right]} \varphi(n g) \psi_{N_{1} p}(n)^{-1} d n
$$

and

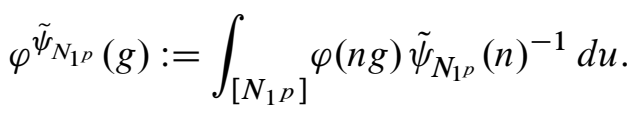

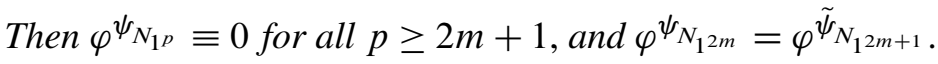




\section{Proof of part (2) of Theorem 2.4}

In this section, we assume that $a \leq 2 m$ and $\sigma$ is $\psi^{\alpha}$-generic for $\alpha \in F^{*} /\left(F^{*}\right)^{2}$, and show that $\mathcal{E}_{\Delta(\tau, b) \otimes \sigma}$ has a nonzero Fourier coefficient attached to $\left[(2 m)(a)^{2 b}\right]$.

First, we construct a residual representation of ${\widetilde{\mathrm{Sp}_{2 a b}}}_{2}(\mathbb{A})$ as follows. For any $\tilde{\phi} \in$ $\mathcal{A}\left(N_{a b}(\mathbb{A}) \tilde{M}_{a b}(F) \backslash \widetilde{\mathrm{Sp}}_{2 a b}(\mathbb{A})\right)_{\gamma_{\psi^{-\alpha}} \Delta(\tau, b)}$, following [Mœglin and Waldspurger 1995], an residual Eisenstein series can be defined by

$$
\tilde{E}(\tilde{\phi}, s)(g)=\sum_{\gamma \in P_{a b}(F) \backslash \mathrm{Sp}_{2 a b}(F)} \lambda_{s} \tilde{\phi}(\gamma g) .
$$

It converges absolutely for real part of $s$ large and has meromorphic continuation to the whole complex plane $\mathbb{C}$. By similar argument as that in [Jiang et al. 2013], this Eisenstein series has a simple pole at $b / 2$, which is the right-most one. Denote the representation generated by these residues at $s=b / 2$ by $\tilde{\mathcal{E}}_{\Delta(\tau, b)}$. This residual representation is square-integrable.

We separate the proof of part (2) of Theorem 2.4 into three steps:

Step (1) $\mathcal{E}_{\Delta(\tau, b) \otimes \sigma}$ has a nonzero Fourier coefficient attached to the partition $\left[(2 m) 1^{2 a b}\right]$ with respect to the character $\psi_{\left[(2 m) 1^{2 a b}\right], \alpha}$ (for definition, see [Jiang and Liu 2015c, Section 2]).

Step (2) $\tilde{\mathcal{E}}_{\Delta(\tau, b)}$ is irreducible. Let $\mathcal{D}_{2 m, \psi^{\alpha}}^{2 a b+2 m}\left(\mathcal{E}_{\Delta(\tau, b) \otimes \sigma}\right)$ be the $\psi^{\alpha}$-descent of $\mathcal{E}_{\Delta(\tau, b) \otimes \sigma}$ [Ginzburg et al. 2011, Section 3.2]. Then, as a representation of $\widetilde{\mathrm{Sp}}_{2 a b}(\mathbb{A})$, it is square-integrable and contains the whole space of the residual representation $\tilde{\mathcal{E}}_{\Delta(\tau, b)}$.

Step (3) $\tilde{\mathcal{E}}_{\Delta(\tau, b)}$ has a nonzero Fourier coefficient attached to the symplectic partition $\left[(a)^{2 b}\right]$.

Proof of part (2) of Theorem 2.4. From the results in steps (1)-(3) above, we can see that $\mathcal{E}_{\Delta(\tau, b+1) \otimes \sigma}$ has a nonzero Fourier coefficient attached to the composite partition $\left[(2 m) 1^{2 a b}\right] \circ\left[(a)^{2 b}\right]$ (for the definition of composite partitions and the attached Fourier coefficients, we refer to [Ginzburg et al. 2003, Section 1]). Therefore, by [Jiang and Liu 2015c, Lemma 3.1] or [Ginzburg et al. 2003, Lemma 2.6], $\mathcal{E}_{\Delta(\tau, b) \otimes \sigma}$ has a nonzero Fourier coefficient attached to $\left[(2 m)(a)^{2 b}\right]$, which completes the proof of the part (2) of Theorem 2.4.

5A. Proof of step (1). Note that by [Ginzburg et al. 2003, Lemma 1.1], $\mathcal{E}_{\Delta(\tau, b) \otimes \sigma}$ has a nonzero Fourier coefficient attached to the partition $\left[(2 m) 1^{2 a b}\right]$ with respect to the character $\psi_{\left[(2 m) 1^{2 a b}\right], \alpha}$ if and only if the $\psi^{\alpha}$-descent $\mathcal{D}_{2 m, \psi^{\alpha}}^{2 a b+2 m}\left(\mathcal{E}_{\Delta(\tau, b) \otimes \sigma}\right)$ of $\mathcal{E}_{\Delta(\tau, b) \otimes \sigma}$ is not identically zero as a representation of $\widetilde{\operatorname{Sp}}_{2 a b}(\mathbb{A})$.

Recall that $P_{r}^{2 l}=M_{r}^{2 l} N_{r}^{2 l}$ (with $1 \leq r \leq l$ ) is the standard parabolic subgroup of $\mathrm{Sp}_{2 l}$ with Levi part $M_{r}^{2 l}$ isomorphic to $\mathrm{GL}_{r} \times \mathrm{Sp}_{2 l-2 r}$ and $N_{r}^{2 l}$ the unipotent radical. $\tilde{P}_{r}^{2 l}(\mathbb{A})$ is the preimage of $P_{r}^{2 l}(\mathbb{A})=\tilde{M}_{r}^{2 l}(\mathbb{A}) N_{r}^{2 l}(\mathbb{A})$ in $\widetilde{\mathrm{Sp}}_{2 l}(\mathbb{A})$. 
Take any $\xi \in \mathcal{E}_{\Delta(\tau, b) \otimes \sigma}$; we will calculate the constant term of the Fourier-Jacobi coefficient $\mathcal{F J}_{\psi_{m-1}^{\alpha}}^{\phi}(\xi)$ along $P_{r}^{2 a b}$, which is denoted by $\mathcal{C}_{N_{r}^{2 a b}}\left(\mathcal{F J}_{\psi_{m-1}^{\alpha}}^{\phi}(\xi)\right)$, where $1 \leq r \leq a b$.

By [Ginzburg et al. 2011, Theorem 7.8],

$$
\begin{aligned}
\mathcal{C}_{N_{r}^{2 a b}}\left(\mathcal{F J}_{\psi_{m-1}^{\alpha}}^{\phi}(\xi)\right) & \sum_{\substack{0 \leq k \leq r \\
\gamma \in P_{r-k, 1^{k}}^{1}(F) \backslash \mathrm{GL}_{r}(F)}} \int_{L(\mathbb{A})} \phi_{1}(i(\lambda)) \mathcal{F J}_{\psi_{m-1+k}^{\alpha}}^{\phi}\left(\mathcal{C}_{N_{r-k}^{2 a b+2 m}}(\xi)\right)(\hat{\gamma} \lambda \beta) d \lambda .
\end{aligned}
$$

We explain the notation used in (5-1) as follows: $N_{r-k}^{2 a b+2 m}$ denotes the unipotent radical of the parabolic subgroup $P_{r-k}^{2 a b+2 m}$ of $\mathrm{Sp}_{2 a b+2 m}$ with the Levi subgroup $\mathrm{GL}_{r-k} \times \mathrm{Sp}_{2 a b+2 m-2 r+2 k}$, and $P_{r-k, 1^{k}}^{1}$ is a subgroup of $\mathrm{GL}_{r}$ consisting of matrices of the form

$$
\left(\begin{array}{ll}
g & x \\
0 & z
\end{array}\right)
$$

with $z \in U_{k}$, the standard maximal unipotent subgroup of $\mathrm{GL}_{k}$. For $g \in \mathrm{GL}_{j}$, with $j \leq a b+m, \hat{g}=\operatorname{diag}\left(g, I_{2 a b+2 m-2 j}, g^{*}\right)$, and $L$ is a unipotent subgroup, consisting of matrices of the form

$$
\lambda=\left(\begin{array}{cc}
I_{r} & 0 \\
x & I_{m}
\end{array}\right)^{\wedge}
$$

with $i(\lambda)$ in the last row of $x$, and

$$
\beta=\left(\begin{array}{cc}
0 & I_{r} \\
I_{m} & 0
\end{array}\right)^{\wedge}
$$

We assume that $\phi=\phi_{1} \otimes \phi_{2}$, with $\phi_{1} \in \mathcal{S}\left(\mathbb{A}^{r}\right)$ and $\phi_{2} \in \mathcal{S}\left(\mathbb{A}^{a b-r}\right)$. Finally, the Fourier-Jacobi coefficients satisfy the identity

$$
\mathcal{F} \mathcal{J}_{\psi_{m-1+k}^{\alpha}}^{\phi_{2}}\left(\mathcal{C}_{N_{r-k}^{2 a b+2 m}}^{2 a}(\xi)\right)(\hat{\gamma} \lambda \beta):=\mathcal{F J}_{\psi_{m-1+k}^{\alpha}}^{\phi_{2}}\left(\mathcal{C}_{N_{r-k}^{2 a b+2 m}}(\rho(\hat{\gamma} \lambda \beta) \xi)\right)(I),
$$

with $\rho(\hat{\gamma} \lambda \beta)$ denoting the right translation by $\hat{\gamma} \lambda \beta$, where the function is regarded as taking first the constant term $\mathcal{C}_{N_{r-k} 2 a b+2 m}(\rho(\hat{\gamma} \lambda \beta) \xi)$, and then after restricted to $\mathrm{Sp}_{2 a b+2 m-2 r+2 k}(\mathbb{A})$, taking the Fourier-Jacobi coefficient

$$
\mathcal{F} \mathcal{J}_{\psi_{m-1+k}^{\alpha}}^{\phi_{2}}
$$

which is a map taking automorphic forms on $\mathrm{Sp}_{2 a b+2 m-2 r+2 k}(\mathbb{A})$ to those on $\widetilde{\mathrm{Sp}}_{2 a b-2 r}(\mathbb{A})$.

By the cuspidal support of $\xi, \mathcal{C}_{N_{r-k}^{2 a b+2 m}}(\xi)$ is identically zero, unless $k=r$ or $r-k=l a$ with $1 \leq l \leq b$. When $k=r$, since $\left[(2 m+2 r) 1^{2 a b-2 r}\right]$ is bigger than $\eta_{\mathfrak{s o}_{2 n+1}}(\mathbb{C}), \mathfrak{s p}_{2 n}(\mathbb{C})(\underline{p}(\psi))$ under the lexicographical ordering, by [Jiang and Liu 2015a, Proposition 6.4; Ginzburg et al. 2003, Lemma 1.1], $\mathcal{F J}_{\psi_{m-1+r}^{\alpha}}^{\phi_{2}}(\xi)$ is 
identically zero, and hence the corresponding term is zero. When $r-k=l a$, with $1 \leq l \leq b$ and $1 \leq k \leq r$, then by Lemma 4.1, after restricting to $\operatorname{Sp}_{2 a(b-l)+2 m}(\mathbb{A})$, $\mathcal{C}_{N_{r-k}^{2 a b+2 m}}(\rho(\hat{\gamma} \lambda \beta) \xi)$ becomes a form in $\mathcal{E}_{\Delta(\tau, b-l) \otimes \sigma}$ whose Arthur parameter is

$$
\psi^{\prime}=(\tau, 2 b-2 l+1) \boxplus \underset{i=2}{r}\left(\tau_{i}, 1\right) .
$$

Since $\left[(2 m+2 k) 1^{2 a(b-l)-2 k}\right]$ is bigger than $\eta_{\mathfrak{s o}_{2 n^{\prime}+1}(\mathbb{C}), \mathfrak{s p}_{2 n^{\prime}}(\mathbb{C})}\left(\underline{p}\left(\psi^{\prime}\right)\right)$ under the lexicographical ordering, where $2 n^{\prime}=2 a(b-l)+2 m$, by [Jiang and Liu 2015a, Proposition 6.4; Ginzburg et al. 2003, Lemma 1.1], it follows that

$$
\mathcal{F J}_{\psi_{m-1+k}^{\alpha}}^{\phi_{2}}\left(\mathcal{C}_{N_{r-k}^{2 a b+2 m}}^{2 a}(\rho(\hat{\gamma} \lambda \beta) \xi)\right)
$$

is also identically zero, and hence the corresponding term is also zero. Therefore, the only possibilities that

$$
\mathcal{C}_{N_{r}^{2 a b}}\left(\mathcal{F J}_{\psi_{m-1}^{\alpha}}^{\phi}(\xi)\right) \neq 0
$$

are $r=l a$ with $1 \leq l \leq b$, and $k=0$. To prove that $\mathcal{F J}_{\psi_{m-1}^{\alpha}}^{\phi}(\xi)$ is not identically zero, we just have to show that

$$
\mathcal{C}_{N_{r}^{2 a b}}\left(\mathcal{F} \mathcal{J}_{\psi_{m-1}^{\alpha}}^{\phi}(\xi)\right) \neq 0 \text { for some } r
$$

Let $r=a b$; then

$$
\mathcal{C}_{N_{a b}^{2 a b}}\left(\mathcal{F J}_{\psi_{m-1}^{\alpha}}^{\phi}(\xi)\right)=\int_{L(\mathbb{A})} \phi_{1}(i(\lambda)) \mathcal{F} \mathcal{J}_{\psi_{m-1}^{\alpha}}^{\phi_{2}}\left(\mathcal{C}_{N_{a b}^{2 a b+2 m}}(\xi)\right)(\lambda \beta) d \lambda .
$$

By Lemma 4.1, when restricted to $\mathrm{GL}_{2 a b}(\mathbb{A}) \times \operatorname{Sp}_{2 m}(\mathbb{A})$,

$$
\mathcal{C}_{N_{a b}^{2 a b+2 m}}(\xi) \in \delta_{P_{a b}^{2 a b+2 m}}^{1 / 2}|\operatorname{det}|^{-\frac{b+1}{2}} \Delta(\tau, b) \otimes \sigma .
$$

Clearly, the integral in (5-2) is not identically zero if and only if $\sigma$ is $\psi^{\alpha}$-generic. By assumption, $\sigma$ is $\psi^{\alpha}$-generic, and hence

$$
\mathcal{F} \mathcal{J}_{\psi_{m-1}^{\alpha}}^{\phi}(\xi)
$$

is not identically zero. Therefore, $\mathcal{E}_{\Delta(\tau, b) \otimes \sigma}$ has a nonzero Fourier coefficient attached to the partition $\left[(2 m) 1^{2 a b}\right]$ with respect to the character $\psi_{\left[(2 m) 1^{2 a b}\right], \alpha}$. This completes the proof of step (1).

5B. Proof of step (2). The proof of irreducibility of $\tilde{\mathcal{E}}_{\Delta(\tau, b)}$ is similar to that of $\tilde{\mathcal{E}}_{\Delta(\tau, 1)}$ which is given in the proof of [Ginzburg et al. 2011, Theorem 2.1]. To show the square-integrable residual representation $\tilde{\mathcal{E}}_{\Delta(\tau, b)}$ is irreducible, it suffices to show that at each local place $v$,

$$
\operatorname{Ind}_{\tilde{P}_{a b}\left(F_{v}\right)}^{\tilde{\mathrm{Sp}}_{2 a b}\left(F_{v}\right)} \mu_{\psi_{v}^{-\alpha}} \Delta\left(\tau_{v}, b\right)|\cdot|^{\frac{b}{2}}
$$


has a unique irreducible quotient, where we assume that $\psi \cong \otimes_{v} \psi_{v}, P_{a b}$ is the parabolic subgroup of $\operatorname{Sp}_{2 a b}$ with Levi subgroup isomorphic to $\mathrm{GL}_{a b}$, and $\tilde{P}_{a b}\left(F_{v}\right)$ is the preimage of $P_{a b}\left(F_{v}\right)$ in $\widetilde{\operatorname{Sp}}_{2 a b}\left(F_{v}\right)$. Note that $\Delta\left(\tau_{v}, b\right)$ is the unique irreducible quotient of the following induced representation

$$
\operatorname{Ind}_{Q_{a b}\left(F_{v}\right)}^{\mathrm{GL}_{a b}\left(F_{v}\right)} \tau_{v}|\cdot|^{\frac{b-1}{2}} \otimes \tau_{v}|\cdot|^{\frac{b-3}{2}} \otimes \cdots \otimes \tau_{v}|\cdot|^{\frac{1-b}{2}},
$$

where $Q_{a^{b}}$ is the parabolic subgroup of $\mathrm{GL}_{a b}$ with Levi subgroup isomorphic to $\mathrm{GL}_{a}^{\times b}$. Let $P_{a^{b}}$ be the parabolic subgroup of $\mathrm{Sp}_{2 a b}$ with Levi subgroup isomorphic to $\mathrm{GL}_{a}^{\times b}$, and $\tilde{P}_{a^{b}}\left(F_{v}\right)$ is the preimage of $P_{a^{b}}\left(F_{v}\right)$ in ${\widetilde{\mathrm{Sp}_{2 a b}}}_{2}\left(F_{v}\right)$. We just have to show that the following induced representation has a unique irreducible quotient

$$
\operatorname{Ind} \underset{\tilde{P}_{a b}\left(F_{v}\right)}{\tilde{S p}_{2 a b}\left(F_{v}\right)} \mu_{\psi_{v}^{-\alpha}} \tau_{v}|\cdot|^{\frac{2 b-1}{2}} \otimes \tau_{v}|\cdot|^{\frac{2 b-3}{2}} \otimes \cdots \otimes \tau_{v}|\cdot|^{\frac{1}{2}} .
$$

Since $\tau_{v}$ is generic and unitary, by [Tadić 1986; Vogan 1986], $\tau_{v}$ is fully parabolic, induced from its Langlands data with exponents in the open interval $\left(-\frac{1}{2}, \frac{1}{2}\right)$. Explicitly, we can assume that

$$
\tau_{v} \cong \rho_{1}|\cdot|^{\alpha_{1}} \times \rho_{2}|\cdot|^{\alpha_{2}} \times \cdots \times \rho_{r}|\cdot|^{\alpha_{r}},
$$

where the $\rho_{i}$ are tempered representations, $\alpha_{i} \in \mathbb{R}$, and $\frac{1}{2}>\alpha_{1}>\alpha_{2}>\cdots>\alpha_{r}>-\frac{1}{2}$. Therefore, the induced representation in (5-4) can be written as

$$
\begin{aligned}
& \mu_{\psi_{v}^{-\alpha}} \rho_{1}|\cdot| \frac{2 b-1}{2}+\alpha_{1} \times \rho_{2}|\cdot|^{\frac{2 b-1}{2}+\alpha_{2}} \times \cdots \times \rho_{r}|\cdot|^{\frac{2 b-1}{2}+\alpha_{r}} \\
& \times \rho_{1}|\cdot|^{\frac{2 b-3}{2}+\alpha_{1}} \times \rho_{2}|\cdot|^{\frac{2 b-3}{2}+\alpha_{2}} \times \cdots \times \rho_{r}|\cdot|^{\frac{2 b-3}{2}+\alpha_{r}} \\
& \quad \times \cdots \times \rho_{1}|\cdot|^{\frac{1}{2}+\alpha_{1}} \times \rho_{2}|\cdot|^{\frac{1}{2}+\alpha_{2}} \times \cdots \times \rho_{r}|\cdot|^{\frac{1}{2}+\alpha_{r}} \rtimes 1_{\tilde{\operatorname{Sp}_{0}}}\left(F_{v}\right)
\end{aligned}
$$

Since $\alpha_{i} \in \mathbb{R}$ and $\frac{1}{2}>\alpha_{1}>\alpha_{2}>\cdots>\alpha_{r}>-\frac{1}{2}$, we can easily see that the exponents satisfy

$$
\begin{aligned}
& \frac{2 b-1}{2}+\alpha_{1}>\frac{2 b-1}{2}+\alpha_{2}>\cdots>\frac{2 b-1}{2}+\alpha_{r} \\
&>\frac{2 b-3}{2}+\alpha_{1}>\frac{2 b-3}{2}+\alpha_{2}>\cdots>\frac{2 b-3}{2}+\alpha_{r} \\
& \quad>\cdots>\frac{1}{2}+\alpha_{1}>\frac{1}{2}+\alpha_{2}>\cdots>\frac{1}{2}+\alpha_{r}>0 .
\end{aligned}
$$

By Langlands classification of metaplectic groups (see [Borel and Wallach 2000; Ban and Jantzen 2013]), one can see that the induced representation in (5-4) has a unique irreducible quotient which is the Langlands quotient. This completes the proof of irreducibility of $\tilde{\mathcal{E}}_{\Delta(\tau, b)}$.

To prove the square-integrability of $\mathcal{D}_{2 m, \psi^{\alpha}}^{2 a b+2 m}\left(\mathcal{E}_{\Delta(\tau, b) \otimes \sigma}\right)$, we need to calculate the automorphic exponent attached to the nontrivial constant term considered in 
step (1); $r=a b$, and for definition of automorphic exponent see [Mœglin and Waldspurger 1995, I.3.3]. For this, we need to consider the action of

$$
\bar{g}=\operatorname{diag}\left(g, g^{*}\right) \in \mathrm{GL}_{a b}(\mathbb{A}) \times \widetilde{\operatorname{Sp}}_{0}(\mathbb{A}) .
$$

Since $r=a b$,

$$
\beta=\left(\begin{array}{cc}
0 & I_{a b} \\
I_{m} & 0
\end{array}\right)^{\wedge}
$$

Let

$$
\tilde{g}:=\beta \operatorname{diag}\left(I_{m}, \bar{g}, I_{m}\right) \beta^{-1}=\operatorname{diag}\left(g, I_{2 m}, g^{*}\right) .
$$

Then changing variables in (5-2) via $\lambda \mapsto \tilde{g} \lambda \tilde{g}^{-1}$ will give a Jacobian $|\operatorname{det} g|^{-m}$. On the other hand, by [Ginzburg et al. 2011, Formula (1.4)], the action of $\bar{g}$ on $\phi_{1}$ gives $\gamma_{\psi^{-\alpha}}(\operatorname{det} g)|\operatorname{det} g|^{1 / 2}$. Therefore, $\bar{g}$ acts by $\Delta(\tau, b)(g)$ with character $\delta_{P_{a b}^{2 a b+2 m}}^{1 / 2}(\tilde{g})|\operatorname{det} g|^{-\frac{b+1}{2}}|\operatorname{det} g|^{-m} \gamma_{\psi^{-\alpha}}(\operatorname{det} g)|\operatorname{det} g|^{\frac{1}{2}}$

$$
=\gamma_{\psi^{-\alpha}}(\operatorname{det} g) \delta_{P_{a b}^{2 a b}}^{1 / 2}(\bar{g})|\operatorname{det} g|^{-\frac{b}{2}} .
$$

Therefore, as a function on $\mathrm{GL}_{a b}(\mathbb{A}) \times \widetilde{\mathrm{Sp}}_{0}(\mathbb{A})$,

$$
\mathcal{C}_{N_{a b}^{2 a b}}\left(\mathcal{F J}_{\psi_{m-1}^{\alpha}}^{\phi}(\xi)\right) \in \gamma_{\psi^{-\alpha}} \delta_{P_{a b}^{2 a b}}^{1 / 2}|\operatorname{det}(\cdot)|^{-\frac{b}{2}} \Delta(\tau, b) \otimes 1_{\tilde{\mathrm{Sp}}_{0}(\mathbb{A})} \cdot
$$

Since, the cuspidal exponent of $\Delta(\tau, b)$ is

$$
\left\{\left(\frac{1-b}{2}, \frac{3-b}{2}, \ldots, \frac{b-1}{2}\right)\right\},
$$

the cuspidal exponent of $\mathcal{C}_{N_{a b}^{2 a b}}\left(\mathcal{F J}_{\psi_{m-1}^{\alpha}}^{\phi}(\xi)\right)$ is

$$
\left\{\left(\frac{1-2 b}{2}, \frac{3-2 b}{2}, \ldots,-\frac{1}{2}\right)\right\} \text {. }
$$

Hence, by Langlands square-integrability criterion [Mœglin and Waldspurger 1995, Lemma I.4.11], the automorphic representation $\mathcal{D}_{2 m, \psi^{\alpha}}^{2 a b+2 m}\left(\mathcal{E}_{\Delta(\tau, b) \otimes \sigma}\right)$ is squareintegrable.

From (5-5), it is easy to see that as a representation of $\mathrm{GL}_{a b}(\mathbb{A}) \times \widetilde{\mathrm{Sp}}_{0}(\mathbb{A})$,

$$
\mathcal{C}_{N_{a b}^{2 a b}}\left(\mathcal{D}_{2 m, \psi^{\alpha}}^{2 a b+2 m}\left(\mathcal{E}_{\Delta(\tau, b) \otimes \sigma}\right)\right)=\gamma_{\psi^{-\alpha}} \delta_{P_{a b}^{2 a b}}^{1 / 2}|\operatorname{det}(\cdot)|^{-\frac{b}{2}} \Delta(\tau, b) \otimes 1_{\tilde{\mathrm{Sp}}_{0}(\mathbb{A})} .
$$

From the cuspidal support of the Speh residual representation $\Delta(\tau, b)$ of $\mathrm{GL}_{a b}(\mathbb{A})$, one can now easily see that

$$
\begin{aligned}
& \mathcal{C}_{N_{a^{b}}^{2 a b}}\left(\mathcal{D}_{2 m, \psi^{\alpha}}^{2 a b+2 m}\left(\mathcal{E}_{\Delta(\tau, b) \otimes \sigma}\right)\right) \\
& \quad=\gamma_{\psi^{-\alpha}} \delta_{P_{a^{b}}^{2 a b}}^{1 / 2} \tau|\cdot|^{\frac{1-2 b}{2}} \otimes \tau|\cdot|^{\frac{3-2 b}{2}} \otimes \cdots \otimes \tau|\cdot|^{-\frac{1}{2}} \otimes 1_{\tilde{\mathrm{Sp}}_{0}(\mathrm{~A})},
\end{aligned}
$$

where $N_{a^{b}}^{2 a b}$ is the unipotent radical of the parabolic subgroup $P_{a^{b}}^{2 a b}$ with Levi isomorphic to $\mathrm{GL}_{a}^{\times b}$. By [op. cit., Corollary 3.14(ii)], any noncuspidal irreducible 
summand of $\mathcal{D}_{2 m, \psi^{\alpha}}^{2 a b+2 m}\left(\mathcal{E}_{\Delta(\tau, b) \otimes \sigma}\right)$ must be contained in the space $\tilde{\mathcal{E}}_{\tau \otimes b}, \Lambda$, which is the residual representation generated by residues of the Eisenstein series associated to the induced representation

$$
\operatorname{Ind}_{\tilde{P}_{a^{b}}^{2 a b}(\mathbb{A})}^{\tilde{\mathrm{Sp}_{2 a b}(\mathbb{A})}} \gamma_{\psi^{-\alpha}} \tau|\cdot|^{s_{1}} \otimes \tau|\cdot|^{s_{2}} \otimes \cdots \otimes \tau|\cdot|^{s_{b}},
$$

at the point

$$
\Lambda=\left\{\frac{1-2 b}{2}, \frac{3-2 b}{2}, \ldots, \frac{-1}{2}\right\} .
$$

Since the Speh residual representation $\Delta(\tau, b)$ of $\mathrm{GL}_{a b}(\mathbb{A})$ is irreducible, by taking residues in stages, one can easily see that the space of the residual representation $\tilde{\mathcal{E}}_{\tau \otimes b, \Lambda}$ is exactly identical to that of $\tilde{\mathcal{E}}_{\Delta(\tau, b)}$. Therefore, any noncuspidal irreducible summand of $\mathcal{D}_{2 m, \psi^{\alpha}}^{2 a b+2 m}\left(\mathcal{E}_{\Delta(\tau, b) \otimes \sigma}\right)$ must be contained in the space $\tilde{\mathcal{E}}_{\Delta(\tau, b)}$. Hence, the descent representation $\mathcal{D}_{2 m, \psi^{\alpha}}^{2 a b+2 m}\left(\mathcal{E}_{\Delta(\tau, b) \otimes \sigma}\right)$ has a nontrivial intersection with the space of the residual representation $\tilde{\mathcal{E}}_{\Delta(\tau, b)}$. Since we have seen that $\tilde{\mathcal{E}}_{\Delta(\tau, b)}$ is irreducible, $\mathcal{D}_{2 m, \psi^{\alpha}}^{2 a b+2 m}\left(\mathcal{E}_{\Delta(\tau, b) \otimes \sigma}\right)$ must contain the whole space of the residual representation $\tilde{\mathcal{E}}_{\Delta(\tau, b)}$. This completes the proof of step (2).

5C. Proof of step (3). The proof of the fact that $\tilde{\mathcal{E}}_{\Delta(\tau, b)}$ has a nonzero Fourier coefficient attached to the symplectic partition $\left[(a)^{2 b}\right]$ is very similar to the proof of [Liu 2013a, Theorem 4.2.2], if $a$ is even. The idea is to apply Lemma 3.2 repeatedly and use induction on $b$. Note that the case of $\tilde{\mathcal{E}}_{\Delta(\tau, 1)}$ has already been proved in [Ginzburg et al. 2011, Theorem 8.1]. We omit the details here for this case.

In the following, we assume that $a=2 k+1$ and prove $\tilde{\mathcal{E}}_{\Delta(\tau, b)}$ has a nonzero Fourier coefficient attached to the symplectic partition $p:=\left[(2 k+1)^{2 b}\right]$ by induction on $b$. When $b=1$, it has been proved in [op. cit., Theorem 8.2], we will use similar idea here. Assume that $\tilde{\mathcal{E}}_{\Delta(\tau, b-1)}$ has a nonzero Fourier coefficient attached to the symplectic partition $\left[(2 k+1)^{2 b-2}\right]$.

Take any $\varphi \in \tilde{\mathcal{E}}_{\Delta(\tau, b)}$; its Fourier coefficients attached to $\underline{p}$ are of the following form

$$
\varphi^{\psi_{\underline{p}}}(g)=\int_{\left[V_{\underline{p}, 2}\right]} \varphi(v g) \psi_{\underline{p}}^{-1}(v) d v .
$$

For definitions of the unipotent group $V_{\underline{p}, 2}$ and its character $\psi_{\underline{p}}$, see [Jiang and Liu 2015c, Section 2].

Note that the one-dimensional torus $\mathcal{H}_{\underline{p}}$ defined in [op. cit, (2.1)] has elements of the form

$$
\mathcal{H}_{\underline{p}}(t)=\operatorname{diag}(A(t), A(t), \ldots, A(t)), \quad \text { where } A(t)=\operatorname{diag}\left(t^{2 k}, t^{2 k-2}, \ldots, t^{-2 k}\right),
$$

and there are $2 b$ copies of $A(t)$. Also note that the group $L_{p}(\mathbb{A})$ defined in [op. cit, Section 2] is isomorphic to $\mathrm{GL}_{2 b}^{2 k+1}(\mathbb{A})$, and the stabilizer of the character $\psi_{\underline{p}}$ 
in $L_{\underline{p}}$ is isomorphic to the diagonal embedding $\widetilde{\operatorname{Sp}_{2 b}}(\mathbb{A})$. Let $\iota$ be this diagonal embedding. Let

$$
N=\left\{n(x):=\left(\begin{array}{ccc}
1 & 0 & x \\
0 & I_{2 b-2} & 0 \\
0 & 0 & 1
\end{array}\right)\right\}
$$

Then

$$
\iota(N)=\left\{\iota(n(x))=\left(\begin{array}{ccc}
I_{2 k+1} & 0 & x I_{2 k+1} \\
0 & I_{(2 k+1)(2 b-2)} & 0 \\
0 & 0 & I_{2 k+1}
\end{array}\right)\right\} .
$$

To show the integral in (5-7) is nonvanishing, it suffices to show that the following integral is nonvanishing:

$$
\int_{F \backslash \mathbb{A}} \int_{\left[V_{\underline{p}, 2]}\right.} \varphi(v n(x) g) \psi_{\underline{p}}^{-1}(v) d v d x .
$$

Let $\omega$ be a Weyl element which sends $\mathcal{H}_{p}(t)$ to the torus element

$$
\operatorname{diag}\left(A(t), t^{2 k} I_{2 b-2}, t^{2 k-2} I_{2 b-2}, \ldots, t^{-2 k} I_{2 b-2}, A(t)\right) .
$$

Then $\omega$ has the form $\operatorname{diag}\left(I_{2 k+1}, \omega_{1}, I_{2 k+1}\right)$. Conjugating from left by $\omega$, the integral in (5-9) becomes

$$
\int_{[W]} \varphi(w \omega g) \psi_{W}^{-1}(w) d w,
$$

where $W=\omega V_{\underline{p}, 2} \iota(N) \omega^{-1}$ and $\psi_{W}(w)=\psi_{\underline{p}}\left(\omega^{-1} w \omega\right)$. Then elements of $W$ have the form

$$
w=\left(\begin{array}{ccc}
z_{2 k+1} & q_{1} & q_{2} \\
0 & w^{\prime} & q_{1}^{*} \\
0 & 0 & z_{2 k+1}^{*}
\end{array}\right)\left(\begin{array}{ccc}
I_{2 k+1} & 0 & 0 \\
p_{1} & I_{(2 b-2)(2 k+1)} & 0 \\
p_{2} & p_{1}^{*} & I_{2 k+1}
\end{array}\right),
$$

where $z_{2 k+1} \in V_{2 k+1}$, the standard maximal unipotent subgroup of $\mathrm{GL}_{2 k+1}$; $w^{\prime} \in \omega_{1} V_{\left[(2 k+1)^{2 b-2}\right], 2} \omega_{1}^{-1} ; q_{1} \in M_{(2 k+1) \times((2 b-2)(2 k+1))}$ with certain conditions; $p_{1} \in M_{((2 b-2)(2 m+1)) \times(2 m+1)}$ with certain conditions; $q_{2} \in M_{(2 k+1) \times(2 k+1)}$, symmetric with respect to the secondary diagonal, such that $q_{2}(i, j)=0$ for $1 \leq j<i \leq 2 k+1$, and $q_{2}(1,1)=q_{2}(2,2)=\cdots=q_{2}(2 k+1,2 k+1)$; $p_{2} \in M_{(2 k+1) \times(2 k+1)}$, symmetric with respect to the secondary diagonal, such that $p_{2}(i, j)=0$ for $1 \leq j \leq i \leq 2 k+1$.

Next, as in the proof of Section 4, we apply Lemma 3.2 to fill the zero entries in $q_{1}, q_{2}$ using the nonzero entries in $p_{1}, p_{2}$. Similarly, to proceed, we need to define 
a sequence of one-dimensional root subgroups and put them in a correct order:

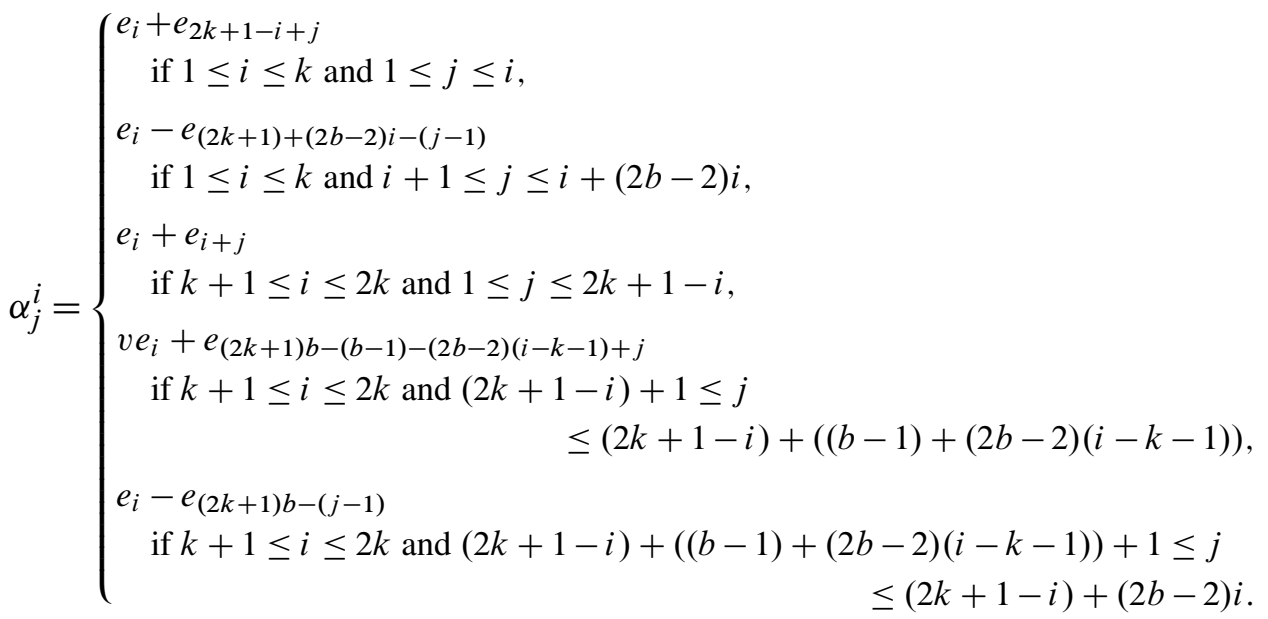

For the above roots $\alpha_{j}^{i}$, let $X_{\alpha_{j}^{i}}$ be the corresponding one-dimensional root subgroup.

For $1 \leq i \leq k$ and $1 \leq j \leq i$, let $\beta_{j}^{i}=-e_{2 k+1-i+j}-e_{i+1}$. For $1 \leq i \leq k$ and $i+1 \leq j \leq i+(2 b-2) i$, let $\beta_{j}^{i}=e_{(2 k+1)+(2 b-2) i-(j-1)}-e_{i+1}$. For $k+1 \leq i \leq 2 k$ and $1 \leq j \leq 2 k+1-i$, let $\beta_{j}^{i}=-e_{i+j}-e_{i+1}$. For $k+1 \leq i \leq 2 k$ and $(2 k+1-i)+1 \leq j \leq(2 k+1-i)+((b-1)+(2 b-2)(i-k-1))$, let $\beta_{j}^{i}=-e_{(2 k+1) b-(b-1)-(2 b-2)(i-k-1)+j}-e_{i+1}$. Finally, for $k+1 \leq i \leq 2 k$ and $(2 k+1-i)+((b-1)+(2 b-2)(i-k-1))+1 \leq j \leq(2 k+1-i)+(2 b-2) i$, let $\beta_{j}^{i}=e_{(2 k+1) b-(j-1)}-e_{i+1}$. For the above roots $\beta_{j}^{i}$, let $X_{\beta_{j}^{i}}$ be the corresponding one-dimensional root subgroup.

Let

$$
m_{i}= \begin{cases}i+(2 b-2) i & \text { if } 1 \leq i \leq k, \\ (2 k+1-i)+(2 b-2) i & \text { if } k+1 \leq i \leq 2 k .\end{cases}
$$

Let $\widetilde{W}$ be the subgroup of $W$ with elements of the form as in (5-11), but with the $p_{1}$ and $p_{2}$ parts zero. Let $\psi_{\widetilde{W}}=\psi_{W} \mid \widetilde{W}$. For any subgroup of $W$ containing $\widetilde{W}$, we automatically extend $\psi_{\widetilde{W}}$ trivially to this subgroup and still denote the character by $\psi_{\widetilde{W}}$.

Next, we will apply Lemma 3.2 to a sequence of quadruples. For any $i$ such that $1 \leq i \leq k+1$, one can see that the following quadruple satisfies all the conditions for Lemma 3.2:

$$
\left(\widetilde{W}_{i}, \psi_{\widetilde{W}},\left\{X_{\alpha_{j}^{i}}\right\}_{j=1}^{m_{i}},\left\{X_{\beta_{j}^{i}}\right\}_{j=1}^{m_{i}}\right),
$$

where

$$
\widetilde{W}_{i}=\prod_{s=1}^{i-1} \prod_{j=1}^{m_{s}} X_{\alpha_{j}^{s}} \widetilde{W} \prod_{l=i+1}^{2 k} \prod_{j=1}^{m_{l}} X_{\beta_{j}^{l}} .
$$


Applying Lemma 3.2, one can see that the integral in (5-10) is nonvanishing if and only if the following integral is nonvanishing:

$$
\int_{\left[\widetilde{W}_{i}^{\prime}\right]} \varphi(w \omega g) \psi{\widetilde{W_{i}^{\prime}}}_{i}^{-1}(w) d w,
$$

where

$$
\widetilde{W}_{i}^{\prime}=\prod_{s=1}^{i} \prod_{j=1}^{m_{s}} X_{\alpha_{j}^{s}} \widetilde{W} \prod_{l=i+1}^{2 k} \prod_{j=1}^{m_{l}} X_{\beta_{j}^{l}},
$$

and $\psi_{\widetilde{W}_{i}^{\prime}}$ is extended from $\psi_{\widetilde{W}}$ trivially.

For any $i$ such that $k+2 \leq i \leq 2 k$, before applying Lemma 3.2 repeatedly to certain sequence of quadruples as above, we need to take the Fourier expansion of the resulting integral at the end of the step $i-1$ along $X_{e_{i}+e_{i}}$ (at the end of step $k+1$, one gets the integral in (5-12) with $i=k+1$ there, at the end of step $s$, $k+2 \leq s \leq 2 k-1$, one would get the integral in (5-14)). Under the action of $\mathrm{GL}_{1}$, we get two kinds of Fourier coefficients corresponding to the two orbits of the dual of $\left[X_{e_{i}+e_{i}}\right]$ : the trivial one and the nontrivial one. It turns out that any Fourier coefficient corresponding to the nontrivial orbit contains an inner integral which is exactly the Fourier coefficients attached to the partition $\left[(2 i) 1^{(2 k+1)(2 b)-2 i}\right]$, which is identically zero by [Jiang and Liu 2015a, Proposition 6.4], since $i \geq k+2$. Therefore only the Fourier coefficient attached to the trivial orbit survives.

After taking Fourier expansion of the resulting integral at the end of step $i-1$ along $X_{e_{i}+e_{i}}$ as above, one can see that the following quadruple satisfies all the conditions for Lemma 3.2:

$$
\left(X_{e_{i}+e_{i}} \widetilde{W}_{i}, \psi_{\widetilde{W}},\left\{X_{\alpha_{j}^{i}}\right\}_{j=1}^{m_{i}},\left\{X_{\beta_{1}^{i}}\right\}_{j=1}^{m_{i}}\right),
$$

where

$$
\widetilde{W}_{i}=\prod_{s=1}^{i-1} \prod_{j=1}^{m_{s}} X_{\alpha_{j}^{s}} \prod_{t=k+2}^{i-1} X_{e_{t}+e_{t}} \widetilde{W} \prod_{l=i+1}^{2 k} \prod_{j=1}^{m_{l}} X_{\beta_{j}^{l}} .
$$

Applying Lemma 3.2, we can see that the resulting integral at the end of step $i-1$ is nonvanishing if and only if the following integral is nonvanishing:

$$
\int_{\left[\widetilde{W}_{i}^{\prime}\right]} \varphi(w \omega g) \psi{\widetilde{W_{i}^{\prime}}}^{-1}(w) d w,
$$

where

$$
\widetilde{W}_{i}^{\prime}=\prod_{s=1}^{i} \prod_{j=1}^{m_{s}} X_{\alpha_{j}^{s}} \prod_{t=k+2}^{i} X_{e_{t}+e_{t}} \widetilde{W} \prod_{l=i+1}^{2 k} \prod_{j=1}^{m_{l}} X_{\beta_{j}^{l}},
$$

and $\psi_{\widetilde{W}_{i}^{\prime}}$ is the trivial extension of $\psi_{\widetilde{W}}$. 
One can see that elements of $\widetilde{W}_{2 k}^{\prime}$ have the following form:

$$
w=\left(\begin{array}{ccc}
z_{2 k+1} & q_{1} & q_{2} \\
0 & w^{\prime} & q_{1}^{*} \\
0 & 0 & z_{2 k+1}^{*}
\end{array}\right)
$$

where $z_{2 k+1} \in V_{2 k+1}$, which is the standard maximal unipotent subgroup of $\mathrm{GL}_{2 k+1}$;

$$
w^{\prime} \in \omega_{1} V_{\left[(2 k+1)^{2 b-2}\right], 2} \omega_{1}^{-1}
$$

$q_{1} \in \operatorname{Mat}_{(2 \mathrm{k}+1) \times(2 \mathrm{k}+1)(2 \mathrm{~b}-2)}$ with $q_{1}(2 k+1, j)=0$ for $1 \leq j \leq(2 k+1)(2 b-2)$; $q_{2} \in \operatorname{Mat}_{(2 k+1) \times(2 k+1)}$, symmetric with respect to the secondary diagonal, with $q_{2}(2 k+1,1)=0$. For $w \in \widetilde{W}_{2 k}^{\prime}$ of form in $(5-16)$,

$$
\psi{\widetilde{W_{2 k}^{\prime}}}^{\prime}(w)=\psi\left(\sum_{i=1}^{2 k} z_{i, i+1}\right) \psi_{\left[(2 k+1)^{2 b-2}\right]}\left(\omega_{1}^{-1} w^{\prime} \omega_{1}\right) .
$$

Now consider the Fourier expansion of the integral in (5-14) along the onedimensional root subgroup $X_{2 e_{2 k+1}}$. By the same reason as above, only the Fourier coefficient corresponding to the trivial orbit of the dual of $\left[X_{2 e_{2 k+1}}\right]$ survives, which is actually equal to the integral in (5-14) (with $i=2 k$ there):

$$
\int_{\left[W_{2 k+1}\right]} \varphi(w \omega g) \psi_{W_{2 k+1}}(w)^{-1} d w
$$

where elements in $W_{2 k+1}$ have the same structure as in (5-16), except that the element $q_{2}(2 k+1,1)$ is not identically zero.

One can see that the integral in (5-17) has an inner integral which is exactly $\varphi^{\psi_{N_{12 k}}}$, using notation in Lemma 5.2 below. On the other hand, we know that by

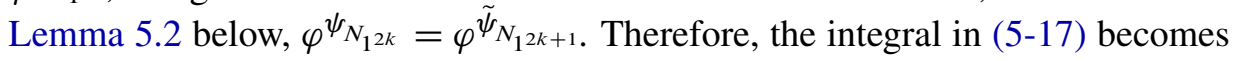

$$
\int_{\left[W_{2 k+1}^{\prime}\right]} \varphi(w \omega g) \psi_{W_{2 k+1}^{\prime}}(w)^{-1} d w
$$

where any element in $W_{2 k+1}^{\prime}$ has the following form:

$$
w=w\left(z_{2 k+1}, w^{\prime}, q_{1}, q_{2}\right)=\left(\begin{array}{ccc}
z_{2 k+1} & q_{1} & q_{2} \\
0 & w^{\prime} & q_{1}^{*} \\
0 & 0 & z_{2 k+1}^{*}
\end{array}\right),
$$

where $z_{2 k+1} \in V_{2 k+1}$, the standard maximal unipotent subgroup of $\mathrm{GL}_{2 k+1}$; $w^{\prime} \in \omega_{1} V_{\left[(2 k+1)^{2 b-2}\right], 2} \omega_{1}^{-1} ; q_{1} \in \operatorname{Mat}_{(2 \mathrm{k}+1) \times(2 \mathrm{k}+1)(2 \mathrm{~b}-2)} ; q_{2} \in \operatorname{Mat}_{(2 k+1) \times(2 k+1)}$, symmetric with respect to the secondary diagonal. For $w \in W_{2 k+1}^{\prime}$ as above,

$$
\psi_{W_{2 k+1}^{\prime}}(w)=\psi\left(\sum_{i=1}^{2 k} z_{i, i+1}\right) \psi_{\left[(2 k+1)^{2 b-2}\right]}\left(\omega_{1}^{-1} w^{\prime} \omega_{1}\right) .
$$


Hence, the integral in (5-18) can be written as

$$
\int_{W_{2 k+1}^{\prime \prime}} \varphi_{P_{2 k+1}}(w \omega g) \psi_{W_{2 k+1}^{\prime \prime}}(w)^{-1} d w
$$

where $W_{2 k+1}^{\prime \prime}$ is a subgroup of $W_{2 k+1}^{\prime}$ consisting only of elements of the form $w\left(z_{2 k+1}, w^{\prime}, 0,0\right)$,

$$
\psi_{W_{2 k+1}^{\prime \prime}}=\left.\psi_{W_{2 k+1}^{\prime}}\right|_{W_{2 k+1}^{\prime \prime}},
$$

and $\varphi_{P_{2 m+1}}$ is the constant term of $\varphi$ along the parabolic subgroup $\tilde{P}_{2 k+1}(\mathbb{A})=$ $\tilde{M}_{2 k+1}(\mathbb{A}) N_{2 k+1}(\mathbb{A})$ of $\widetilde{\mathrm{Sp}}_{2 b(2 k+1)}(\mathbb{A})$ with the Levi subgroup isomorphic to $\mathrm{GL}_{2 k+1}(\mathbb{A}) \times \widetilde{\mathrm{Sp}}_{(2 b-2)(2 k+1)}(\mathbb{A})$.

By Lemma 5.1 below, $\varphi(w \omega g)_{\tilde{P}_{2 k+1}(\mathbb{A})}$ is an automorphic form in

$$
\gamma_{\psi^{-\alpha}} \tau|\cdot|^{-\frac{2 b-1}{2}} \otimes \tilde{\mathcal{E}}_{\Delta(\tau, b-1)}
$$

when restricted to the Levi subgroup. Note that the restriction of $\psi_{W_{2 k+1}^{\prime}}$ to the $z_{2 k+1}$-part gives us a Whittaker coefficient of $\tau$, and the restriction to the $w^{\prime}$-part gives a Fourier coefficient of $\tilde{\mathcal{E}}_{\Delta(\tau, b-1)}$ attached to the partition $\left[(2 k+1)^{2 b-2}\right]$, up to the conjugation of the Weyl element $\omega_{1}$. On the other hand, $\tau$ is generic, and by induction assumption, $\tilde{\mathcal{E}}_{\Delta(\tau, b-1)}$ has a nonzero Fourier coefficient attached to the partition $\left[(2 k+1)^{2 b-2}\right]$. Therefore, we can conclude that $\tilde{\mathcal{E}}_{\Delta(\tau, b)}$ has a nonzero $\psi_{p}$-Fourier coefficient attached to the partition $\left[(2 k+1)^{2 b}\right]$. This completes the proof of step (3), up to Lemmas 5.1 and 5.2, which are stated below.

We remark that as Lemmas 4.1 and 4.2, Lemmas 5.1 and 5.2 below are also analogues of [Liu 2013a, Lemmas 4.2.4 and 4.2.6], with similar arguments, and hence we again only state them without proofs.

Lemma 5.1. Let $\tilde{P}_{(2 k+1) i}(\mathbb{A})=\tilde{M}_{(2 k+1) i}(\mathbb{A}) N_{(2 k+1) i}(\mathbb{A})$, with $1 \leq i \leq b$, be the parabolic subgroup of $\widetilde{\mathrm{Sp}}_{2 b(2 k+1)}(\mathbb{A})$ with Levi part

$$
\tilde{M}_{(2 k+1) i}(\mathbb{A}) \cong \mathrm{GL}_{(2 k+1) i}(\mathbb{A}) \times \widetilde{\operatorname{Sp}}_{(2 k+1)(2 b-2)}(\mathbb{A}) .
$$

Let $\varphi$ be an arbitrary automorphic form in $\tilde{\mathcal{E}}_{\Delta(\tau, b)}$. Denote by $\varphi_{P_{(2 k+1) i}}$ the constant term of $\varphi$ along $P_{(2 k+1) i}$. Then, for $1 \leq i \leq b, \varphi_{P_{(2 k+1) i}}$ belongs to

$$
\mathcal{A}\left(N_{(2 k+1) i}(\mathbb{A}) \tilde{M}_{(2 k+1) i}(F) \backslash \widetilde{\operatorname{Sp}}_{2 b(2 k+1)}(\mathbb{A})\right)_{\gamma_{\psi-\alpha} \Delta(\tau, i)|\cdot|^{-(2 b-i) / 2} \otimes \tilde{\mathcal{E}}_{\Delta(\tau, b-i)}} .
$$

Lemma 5.2. Let $N_{1^{p}}(\mathbb{A})$ be the unipotent radical of the parabolic subgroup $\tilde{P}_{1^{p}}(\mathbb{A})$ of $\widetilde{\mathrm{Sp}}_{2 b(2 k+1)}(\mathbb{A})$ with Levi part isomorphic to

$$
\mathrm{GL}_{1}^{\times p}(\mathbb{A}) \times \widetilde{\mathrm{Sp}}_{2 b(2 k+1)-2 p}(\mathbb{A}) .
$$

Let

$$
\psi_{N_{1} p}(n):=\psi\left(n_{1,2}+\cdots+n_{p, p+1}\right) \quad \text { and } \quad \tilde{\psi}_{N_{1 p}}(n):=\psi\left(n_{1,2}+\cdots+n_{p-1, p}\right) \text {, }
$$


be two characters of $N_{1^{p}}(\mathbb{A})$. For any automorphic form $\varphi \in \tilde{\mathcal{E}}_{\Delta(\tau, b)}$, define $\psi_{N_{1^{p}}}$ and $\tilde{\psi}_{N_{1} p}$-Fourier coefficients by:

$$
\begin{aligned}
& \varphi^{\psi_{N_{1} p}}(g):=\int_{\left[N_{1^{p}}\right]} \varphi(n g) \psi_{N_{1^{p}}}(n)^{-1} d n, \\
& \varphi^{\tilde{\psi}_{N_{1^{p}}}(g)}:=\int_{\left[N_{1^{p}}\right]} \varphi(n g) \tilde{\psi}_{N_{1^{p}}}(n)^{-1} d u .
\end{aligned}
$$

Then $\varphi^{\psi_{N_{1} p}} \equiv 0$ for all $p \geq 2 k+1$, and $\varphi^{\psi_{N_{12 k}}}=\varphi^{\tilde{\psi}_{N_{12 k+1}}}$.

\section{Proof of Theorem 2.6}

In this section, we prove that $\mathcal{E}_{\Delta(\tau, b+1) \otimes \sigma}$ has a nonzero Fourier coefficient attached to $\left[(2 m+2 a)(a)^{2 b}\right]$. Assume that $\sigma$ is $\psi^{\alpha}$-generic with $\alpha \in F^{*} /\left(F^{*}\right)^{2}$.

As in the proof of part (2) of Theorem 2.4 in Section 5 we separate the proof of Theorem 2.6 into two steps:

Step (1) $\mathcal{E}_{\Delta(\tau, b+1) \otimes \sigma}$ has a nonzero Fourier coefficient attached to the partition $\left[(2 m+2 a) 1^{2 a b}\right]$ with respect to the character $\psi_{\left[(2 m+2 a) 1^{2 a b}\right], \alpha}$ (for the definition, see [Jiang and Liu 2015c, Section 2]).

Step (2) Let

$$
\mathcal{D}_{2 m, \psi^{\alpha}}^{2 a(b+1)+2 m}\left(\mathcal{E}_{\Delta(\tau, b+1) \otimes \sigma}\right)
$$

be the $\psi^{\alpha}$-descent from the representation $\mathcal{E}_{\Delta(\tau, b+1) \otimes \sigma}$ of $\operatorname{Sp}_{2 a(b+1)+2 m}(\mathbb{A})$ to a representation of $\widetilde{\mathrm{Sp}}_{2 a b}(\mathbb{A})$. Then it is square-integrable and contains the whole space of the residual representation $\tilde{\mathcal{E}}_{\Delta(\tau, b)}$ which is irreducible and constructed at the beginning of Section 5 .

Proof of Theorem 2.6. First, recall from the step (3) in the proof of part (2) of Theorem 2.4 that $\tilde{\mathcal{E}}_{\Delta(\tau, b)}$ has a nonzero Fourier coefficient attached to the symplectic partition $\left[(a)^{2 b}\right]$. From the results in steps (1) and (2) above, we can see that $\mathcal{E}_{\Delta(\tau, b+1) \otimes \sigma}$ has a nonzero Fourier coefficient attached to the composite partition $\left[(2 m+2 a) 1^{2 a b}\right] \circ\left[(a)^{2 b}\right]$ (for the definition of composite partitions and the attached Fourier coefficients, we refer to [Ginzburg et al. 2003, Section 1]). Therefore, by [Jiang and Liu 2015c, Lemma 3.1] or [Ginzburg et al. 2003, Lemma 2.6], $\mathcal{E}_{\Delta(\tau, b+1) \otimes \sigma}$ has a nonzero Fourier coefficient attached to $\left[(2 m+2 a)(a)^{2 b}\right]$.

Before proving the above two steps, we record the following lemma which is analogous to Lemma 4.1, whose proof will be omitted.

Lemma 6.1. Let $P_{a i}=M_{a i} N_{a i}$, with $1 \leq i \leq b+1$, be the parabolic subgroup of $\mathrm{Sp}_{2 a(b+1)+2 m}$ whose Levi part $M_{a i} \cong \mathrm{GL}_{a i} \times \mathrm{Sp}_{a(2 b+2-2 i)+2 m}$. Let $\varphi$ be an arbitrary automorphic form in $\mathcal{E}_{\Delta(\tau, b+1) \otimes \sigma}$. Denote by $\varphi_{P_{a i}}(g)$ the constant term 
of $\varphi$ along $P_{a i}$. Then, for $1 \leq i \leq b+1$,

$\varphi_{P_{a i}} \in \mathcal{A}\left(N_{a i}(\mathbb{A}) M_{a i}(F) \backslash \operatorname{Sp}_{2 a(b+1)+2 m}(\mathbb{A})\right)_{\Delta(\tau, i)|\cdot|^{-(2 b+1-i) / 2} \otimes \mathcal{E}_{\Delta(\tau, b+1-i) \otimes \sigma}}$.

Note that when $i=b, \mathcal{E}_{\Delta(\tau, b+1-i) \otimes \sigma}=\mathcal{E}_{\tau \otimes \sigma}$, which is not a residual representation as explained at the end of Section $2 B$, is nonzero and generic by [Shahidi 2010, Theorem 7.1.3]; and when $i=b+1, \mathcal{E}_{\Delta(\tau, b+1-i) \otimes \sigma}=\sigma$.

6A. Proof of step (1). By [Ginzburg et al. 2003, Lemma 1.1], $\mathcal{E}_{\Delta(\tau, b+1) \otimes \sigma}$ has a nonzero Fourier coefficient attached to the partition $\left[(2 m+2 a) 1^{2 a b}\right]$ with respect to the character $\psi_{\left[(2 m+2 a) 1^{2 a b}\right], \alpha}$ if and only if the $\psi^{\alpha}$-descent

$$
\mathcal{D}_{2 m+2 a, \psi^{\alpha}}^{2 a(b+1)+2 m}\left(\mathcal{E}_{\Delta(\tau, b+1) \otimes \sigma}\right)
$$

of $\mathcal{E}_{\Delta(\tau, b+1) \otimes \sigma}$, which is a representation of $\widetilde{\mathrm{Sp}}_{2 a b}(\mathbb{A})$, is not identically zero.

Take any $\xi \in \mathcal{E}_{\Delta(\tau, b+1) \otimes \sigma}$, we will calculate the constant term of

$$
\mathcal{F} \mathcal{J}_{\psi_{m+a-1}^{\alpha}}^{\phi}(\xi)
$$

along the parabolic subgroup $\tilde{P}_{r}^{2 a b}(\mathbb{A})=\tilde{M}_{r}^{2 a b}(\mathbb{A}) N_{r}^{2 a b}(\mathbb{A})$ of $\widetilde{\mathrm{Sp}}_{2 a b}(\mathbb{A})$ with Levi subgroup isomorphic to $\mathrm{GL}_{r}(\mathbb{A}) \times \widetilde{\mathrm{Sp}}_{2 a b-2 r}(\mathbb{A}), 1 \leq r \leq a b$, which is denoted by

$$
\mathcal{C}_{N_{r}^{2 a b}}\left(\mathcal{F J}_{\psi_{m+a-1}^{\alpha}}^{\phi}(\xi)\right) .
$$

By [Ginzburg et al. 2011, Theorem 7.8],

(6-1) $\mathcal{C}_{N_{r}^{2 a b}}\left(\mathcal{F J}_{\psi_{m+a-1}^{\alpha}}^{\phi}(\xi)\right)$

$$
=\sum_{\substack{0 \leq k \leq r \\ \gamma \in P_{r-k, 1^{k}}^{1}(F) \backslash \mathrm{GL}_{r}(F)}} \int_{L(\mathbb{A})} \phi_{1}(i(\lambda)) \mathcal{F J}_{\psi_{m+a-1+k}^{\alpha}}^{\phi_{2}}\left(\mathcal{C}_{N_{r-k}^{2 a(b+1)+2 m}}(\xi)\right)(\hat{\gamma} \lambda \beta) d \lambda,
$$

The notation in (6-1) is explained in order: $N_{r-k}^{2 a(b+1)+2 m}$ is the unipotent radical of the parabolic subgroup $P_{r-k}^{2 a(b+1)+2 m}$ of $\mathrm{Sp}_{2 a(b+1)+2 m} ; P_{r-k, 1^{k}}^{1}$ is a subgroup of $\mathrm{GL}_{r}$ consisting of matrices of the form

$$
\left(\begin{array}{ll}
g & x \\
0 & z
\end{array}\right)
$$

with $z \in U_{k}$, the standard maximal unipotent subgroup of $\mathrm{GL}_{k}$. For $g \in \mathrm{GL}_{j}$, $j \leq a(b+1)+m, \hat{g}=\operatorname{diag}\left(g, I_{2 a}(b+1)+2 m-2 j, g^{*}\right) ; L$ is a unipotent subgroup, consisting of matrices of the form

$$
\lambda=\left(\begin{array}{cc}
I_{r} & 0 \\
x & I_{m+a}
\end{array}\right)^{\wedge},
$$


and $i(\lambda)$ is the last row of $x$, and

$$
\beta=\left(\begin{array}{cc}
0 & I_{r} \\
I_{m+a} & 0
\end{array}\right)^{\wedge}
$$

Finally, the Schwartz function $\phi=\phi_{1} \otimes \phi_{2}$ with $\phi_{1} \in \mathcal{S}\left(\mathbb{A}^{r}\right)$ and $\phi_{2} \in \mathcal{S}\left(\mathbb{A}^{a b-r}\right)$, and the function

$$
\begin{aligned}
\mathcal{F J}_{\psi_{m+a-1+k}^{\alpha}}^{\phi_{2}}\left(\mathcal{C}_{\left.N_{r-k}^{2 a(b+1)+2 m}(\xi)\right)(\hat{\gamma} \lambda \beta)}\right. & :=\mathcal{F J}_{\psi_{m+a-1+k}^{\alpha}}^{\phi_{2}}\left(\mathcal{C}_{N_{r-k}^{2 a(b+1)+2 m}}^{2 a}(\rho(\hat{\gamma} \lambda \beta) \xi)\right)(I),
\end{aligned}
$$

with $\rho(\hat{\gamma} \lambda \beta)$ denoting the right translation by $\hat{\gamma} \lambda \beta$, is a composition of the restriction to $\operatorname{Sp}_{2 a(b+1)+2 m-2 r+2 k}(\mathbb{A})$ of $\mathcal{C}_{N_{r-k}^{2 a(b+1)+2 m}}(\rho(\hat{\gamma} \lambda \beta) \xi)$ with the Fourier-Jacobi coefficient

$$
\mathcal{F J}_{\psi_{m+a-1+k}^{\alpha}}^{\phi_{2}}
$$

which takes automorphic forms on $\mathrm{Sp}_{2 a(b+1)+2 m-2 r+2 k}(\mathbb{A})$ to those forms on $\widetilde{\mathrm{Sp}}_{2 a b-2 r}(\mathbb{A})$.

By the cuspidal support of $\xi$,

$$
\mathcal{C}_{N_{r-k}^{2 a(b+1)+2 m}(\xi)}
$$

is identically zero, unless $k=r$ or $r-k=l a$ with $1 \leq l \leq b+1$. When $k=r$, since $\left[(2 m+2 a+2 r) 1^{2 a b-2 r}\right]$ is bigger than $\eta_{\mathfrak{s o}_{2 n+1}(\mathbb{C}), \mathfrak{s p}_{2 n}(\mathbb{C})}(\underline{p}(\psi))$ under the lexicographical ordering, by [Jiang and Liu 2015a, Proposition 6.4; Ginzburg et al. 2003, Lemma 1.1],

$$
\mathcal{F} \mathcal{J}_{\psi_{m+a-1+r}^{\alpha}}^{\phi_{2}}(\xi)
$$

is identically zero, hence the corresponding term is zero. When $r-k=l a$, $1 \leq l \leq b+1$ and $1 \leq k \leq r$, by Lemma 6.1, after restricting to $\operatorname{Sp}_{2 a(b+1-l)+2 m}(\mathbb{A})$,

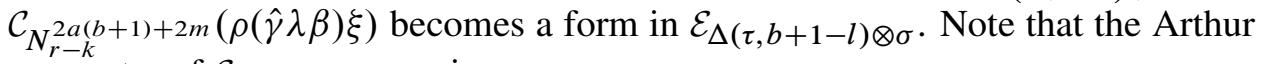
parameter of $\mathcal{E}_{\Delta(\tau, b+1-l) \otimes \sigma}$ is

$$
\psi^{\prime}= \begin{cases}(\tau, 2 b-2 l+1) \boxplus(\tau, 1) \boxplus_{i=3}^{r}\left(\tau_{i}, 1\right) & \text { if } 1 \leq l \leq b, \\ \boxplus_{i=3}^{r}\left(\tau_{i}, 1\right) & \text { if } l=b+1 .\end{cases}
$$

Since $\left[(2 m+2 k) 1^{2 a(b+1-l)-2 k}\right]$ is bigger than $\eta_{\mathfrak{s o}_{2 n^{\prime}+1}(\mathbb{C}), \mathfrak{s p}_{2 n^{\prime}}(\mathbb{C})}\left(\underline{p}\left(\psi^{\prime}\right)\right)$ under the lexicographical ordering, where $2 n^{\prime}=2 a(b+1-l)+2 m$, by [Jiang and Liu 2015a, Proposition 6.4; Ginzburg et al. 2003, Lemma 1.1],

$$
\mathcal{F} \mathcal{J}_{\psi_{m+a-1+k}^{\alpha}}^{\phi_{2}}\left(\mathcal{C}_{\left.N_{r-k}^{2 a(b+1)+2 m}(\rho(\hat{\gamma} \lambda \beta) \xi)\right)}\right.
$$

is also identically zero. Hence the corresponding term is also zero.

It follows that the only possibilities for which

$$
\mathcal{C}_{N_{r}^{2 a(b+1)+2 m}}\left(\mathcal{F J}_{\psi_{m+a-1}^{\alpha}}^{\phi}(\xi)\right) \neq 0
$$


are $r=l a$ with $1 \leq l \leq b+1$, and $k=0$. To prove that $\mathcal{F J}_{\psi_{m+a-1}^{\alpha}}^{\phi}(\xi)$ is not identically zero, we just have to show that

for some $r$.

$$
\mathcal{C}_{N_{r}^{2 a b}}\left(\mathcal{F J}_{\psi_{m+a-1}^{\alpha}}^{\phi}(\xi)\right) \neq 0
$$

Take $r=a b$. Then we have

$$
\begin{aligned}
& \mathcal{C}_{N_{a b}^{2 a b}}\left(\mathcal{F J}_{\psi_{m+a-1}^{\alpha}}^{\phi}(\xi)\right) \\
& =\int_{L(\mathbb{A})} \phi_{1}(i(\lambda)) \mathcal{F J}_{\psi_{m+a-1}^{\alpha}}^{\phi_{2}}\left(\mathcal{C}_{\left.N_{a b}^{2 a(b+1)+2 m}(\xi)\right)(\lambda \beta) d \lambda .}\right.
\end{aligned}
$$

By Lemma 6.1, when restricted to $\mathrm{GL}_{2 a b}(\mathbb{A}) \times \operatorname{Sp}_{2 m+2 a}(\mathbb{A})$,

$$
\mathcal{C}_{N_{a b}^{2 a(b+1)+2 m}}(\xi) \in \delta_{P_{a b}^{2 a(b+1)+2 m}}^{1 / 2}|\cdot|^{-\frac{b+1}{2}} \Delta(\tau, b) \otimes\left(\mathcal{E}_{\tau \otimes \sigma}\right),
$$

where $\mathcal{E}_{\tau \otimes \sigma}$ is not a residual representation as explained at the end of Section 2B.

Clearly, the integral in (6-2) is not identically zero if and only if $\mathcal{E}_{\tau \otimes \sigma}$ is $\psi^{\alpha}$-generic. Since by assumption, $\sigma$ is $\psi^{\alpha}$-generic, we have that $\mathcal{E}_{\tau \otimes \sigma}$ is also $\psi^{\alpha}$-generic by [Shahidi 2010, Theorem 7.1.3]. Hence,

$$
\mathcal{F J}_{\psi_{m+a-1}^{\alpha}}^{\phi}(\xi)
$$

is not identically zero. Therefore, $\mathcal{E}_{\Delta(\tau, b) \otimes \sigma}$ has a nonzero Fourier coefficient attached to the partition $\left[(2 m+2 a) 1^{2 a b}\right]$ with respect to the character

$$
\psi_{\left[(2 m+2 a) 1^{2 a b}\right], \alpha} .
$$

This completes the proof of step (1).

6B. Proof of step (2). To prove the square-integrability of the descent representation

$$
\mathcal{D}_{2 m+2 a, \psi^{\alpha}}^{2 a(b+1)+2 m}\left(\mathcal{E}_{\Delta(\tau, b+1) \otimes \sigma}\right),
$$

as in Section 5B, we need to calculate the automorphic exponent attached to the nontrivial constant term considered in step (1) $(r=a b)$. For this, we need to consider the action of

$$
\bar{g}=\operatorname{diag}\left(g, g^{*}\right) \in \mathrm{GL}_{a b}(\mathbb{A}) \times{\widetilde{\mathrm{Sp}_{0}}}_{(\mathbb{A})} .
$$

Since $r=a b$, we have that $\beta=\left(\begin{array}{cc}0 & I_{a b} \\ I_{m+a} & 0\end{array}\right)$. Let

$$
\tilde{g}:=\beta \operatorname{diag}\left(I_{m+a}, \bar{g}, I_{m+a}\right) \beta^{-1}=\operatorname{diag}\left(g, I_{2 m+2 a}, g^{*}\right) \text {. }
$$

Then changing variables in (5-2), $\lambda \mapsto \tilde{g} \lambda \tilde{g}^{-1}$ will give a Jacobian $|\operatorname{det} g|^{-m-a}$. On the other hand, by [Ginzburg et al. 2011, Formula (1.4)], the action of $\bar{g}$ on $\phi_{1}$ 
gives $\gamma_{\psi^{-\alpha}}(\operatorname{det} g)|\operatorname{det} g|^{\frac{1}{2}}$. Therefore, $\bar{g}$ acts by $\Delta(\tau, b)(g)$ with character

$$
\begin{aligned}
\delta_{P_{a b}^{2 a(b+1)+2 m}}^{1 / 2}(\tilde{g})|\operatorname{det} g|^{-\frac{b+1}{2}}|\operatorname{det} g|^{-m-a} \gamma_{\psi^{-\alpha}} & (\operatorname{det} g)|\operatorname{det} g|^{\frac{1}{2}} \\
& =\gamma_{\psi^{-\alpha}}(\operatorname{det} g) \delta_{P_{a b}^{2 a b}}^{1 / 2}(\bar{g})|\operatorname{det} g|^{-\frac{b}{2}} .
\end{aligned}
$$

Therefore, as a function on $\mathrm{GL}_{a b}(\mathbb{A}) \times \widetilde{\mathrm{Sp}}_{0}(\mathbb{A})$,

$$
\mathcal{C}_{N_{a b}^{2 a b}}\left(\mathcal{F J}_{\psi_{m+a-1}^{\alpha}}^{\phi}(\xi)\right) \in \gamma_{\psi^{-\alpha}} \delta_{P_{a b}^{2 a b}}^{1 / 2}|\operatorname{det}(\cdot)|^{-\frac{b}{2}} \Delta(\tau, b) \otimes 1_{\tilde{\mathrm{Sp}}_{0}(\mathbb{A})} .
$$

Since the cuspidal exponent of $\Delta(\tau, b)$ is

$$
\left\{\left(\frac{1-b}{2}, \frac{3-b}{2}, \ldots, \frac{b-1}{2}\right)\right\},
$$

the cuspidal exponent of $\mathcal{C}_{N_{a b}^{2 a b}}\left(\mathcal{F J}_{\psi_{m+a-1}^{\alpha}}^{\phi}(\xi)\right)$ is

$$
\left\{\left(\frac{1-2 b}{2}, \frac{3-2 b}{2}, \ldots,-\frac{1}{2}\right)\right\} \text {. }
$$

Hence, by the Langlands square-integrability criterion ([Mœglin and Waldspurger 1995, Lemma I.4.11]), the automorphic representation

$$
\mathcal{D}_{2 m+2 a, \psi^{\alpha}}^{2 a(b+1)+2 m}\left(\mathcal{E}_{\Delta(\tau, b+1) \otimes \sigma}\right)
$$

is square integrable.

From (6-3), it follows that as a representation of $\mathrm{GL}_{a b}(\mathbb{A}) \times \widetilde{\mathrm{Sp}}_{0}(\mathbb{A})$,

$$
\begin{aligned}
\mathcal{C}_{N_{a b}^{2 a b}}\left(\mathcal{D}_{2 m+2 a, \psi^{\alpha}}^{2 a(b+1)+2 m}\left(\mathcal{E}_{\Delta(\tau, b+1)} \otimes \sigma\right)\right) & \\
& =\gamma_{\psi^{-\alpha}} \delta_{P_{a b}^{2 a b}}^{1 / 2}|\operatorname{det}(\cdot)|^{-\frac{b}{2}} \Delta(\tau, b) \otimes 1_{\tilde{\operatorname{Sp}}(\mathbb{A})} .
\end{aligned}
$$

Therefore, a similar argument as in Section 5B implies that any noncuspidal summand of

$$
\mathcal{D}_{2 m+2 a, \psi^{\alpha}}^{2 a(b+1)+2 m}\left(\mathcal{E}_{\Delta(\tau, b+1) \otimes \sigma}\right)
$$

must be an irreducible subrepresentation of $\tilde{\mathcal{E}}_{\Delta(\tau, b)}$. Hence,

$$
\mathcal{D}_{2 m+2 a, \psi^{\alpha}}^{2 a(b+1)+2 m}\left(\mathcal{E}_{\Delta(\tau, b+1) \otimes \sigma}\right)
$$

has a nontrivial intersection with the space of the residual representation $\tilde{\mathcal{E}}_{\Delta(\tau, b)}$. Since $\tilde{\mathcal{E}}_{\Delta(\tau, b)}$ is irreducible,

$$
\mathcal{D}_{2 m+2 a, \psi^{\alpha}}^{2 a(b+1)+2 m}\left(\mathcal{E}_{\Delta(\tau, b+1) \otimes \sigma}\right)
$$

must contain the whole space of the residual representation $\tilde{\mathcal{E}}_{\Delta(\tau, b)}$. This completes the proof of step (2). 


\section{Proof of Theorem 2.7}

In this section, assuming that $a=2 k, L\left(\frac{1}{2}, \tau \times \sigma\right) \neq 0, \sigma \not 1_{\mathrm{Sp}_{0}(\mathbb{A})}$, and $\mathcal{E}_{\tau \otimes \sigma}$ has a nonzero Fourier coefficient attached to the partition $[(2 k+2 m)(2 k)]$, we prove that $\mathcal{E}_{\Delta(\tau, b) \otimes \sigma}$ has a nonzero Fourier coefficient attached to the partition $\left[(2 k+2 m)(2 k)^{2 b-1}\right]$, for any $b \geq 1$.

Without loss of generality, by [Jiang and Liu 2015c, Lemma 3.1] or [Ginzburg et al. 2003, Lemma 2.6], we may assume that $\mathcal{E}_{\tau \otimes \sigma}$ has a nonzero Fourier coefficient corresponding to the partition $\left[(2 k+2 m) 1^{2 k}\right]$ with respect to the character $\psi_{\left[(2 k+2 m) 1^{2 k}\right], \alpha}$ for some $\alpha \in F^{*} /\left(F^{*}\right)^{2}$. Then the $\psi^{\alpha}$-descent of $\mathcal{E}_{\tau \otimes \sigma}$ is a generic representation of $\widetilde{\mathrm{Sp}}_{2 k}(\mathbb{A})$. Note that by the constant formula in [Ginzburg et al. 2011, Theorem 7.8], one can easily see that this descent is also a cuspidal representation of $\widetilde{\operatorname{Sp}}_{2 k}(\mathbb{A})$

Similarly as in previous sections, we separate the proof of Theorem 2.7 into three steps:

Step (1) $\mathcal{E}_{\Delta(\tau, b) \otimes \sigma}$ has a nonzero $\psi_{\left[(2 k+2 m) 1^{2 k(2 b-1)}\right], \alpha}$-Fourier coefficient attached to the partition $\left[(2 k+2 m) 1^{2 k(2 b-1)}\right]$ (for definition, see [Jiang and Liu 2015c, Section 2]).

Step (2) Let $\tilde{\sigma}$ be any irreducible subrepresentation of the $\psi^{\alpha}$-descent of $\mathcal{E}_{\tau \otimes \sigma}$. Then it is a generic cuspidal representation of $\widetilde{\operatorname{Sp}}_{2 k}(\mathbb{A})$ which is weakly lifting to $\tau$. Using the theory of theta correspondence and the strong lifting from generic cuspidal representations of $\mathrm{SO}_{2 n+1}(\mathbb{A})$ to automorphic representations of $\mathrm{GL}_{2 n}(\mathbb{A})$, proved in [Jiang and Soudry 2003] (see also [Cogdell et al. 2004]), $\tau$ is also a strong lifting of $\tilde{\sigma}$.

Define a residual representation $\tilde{\mathcal{E}}_{\Delta(\tau, b-1) \otimes \tilde{\sigma}}$ as follows: for any

$$
\tilde{\phi} \in \mathcal{A}\left(N_{k(2 b-1)}(\mathbb{A}) \tilde{M}_{k(2 b-1)}(F) \backslash \widetilde{\operatorname{Sp}}_{2 k(2 b-1)}(\mathbb{A})\right)_{\gamma_{\psi-\alpha} \Delta(\tau, b-1) \otimes \tilde{\sigma}}
$$

one defines as in [Mœglin and Waldspurger 1995]) the residual Eisenstein series

$$
\tilde{E}(\tilde{\phi}, s)(g)=\sum_{\gamma \in P_{k(2 b-1)}(F) \backslash \operatorname{Sp}_{2 k(2 b-1)}(F)} \lambda_{s} \tilde{\phi}(\gamma g) .
$$

It converges absolutely for real part of $s$ large and has meromorphic continuation to the whole complex plane $\mathbb{C}$. By similar argument as that in [Jiang et al. 2013], this Eisenstein series has a simple pole at $b / 2$, which is the right-most one. Denote the representation generated by these residues at $s=b / 2$ by $\tilde{\mathcal{E}}_{\Delta(\tau, b-1) \otimes \tilde{\sigma}}$. This residual representation is square-integrable. Since $\tau$ is also a strong lifting of $\tilde{\sigma}$, the same argument as in Section 5B implies that $\tilde{\mathcal{E}}_{\Delta(\tau, b-1) \otimes \tilde{\sigma}}$ is also irreducible (details will be omitted).

Let

$$
\mathcal{D}_{2 k+2 m, \psi^{\alpha}}^{4 k b+2 m}\left(\mathcal{E}_{\Delta(\tau, b) \otimes \sigma}\right)
$$


be the $\psi^{\alpha}$-descent of $\mathcal{E}_{\Delta(\tau, b) \otimes \sigma}$. Then as a representation of $\widetilde{\operatorname{Sp}}_{2 k(2 b-1)}(\mathbb{A})$, it is square-integrable and contains the whole space of the residual representation $\tilde{\mathcal{E}}_{\Delta(\tau, b-1) \otimes \tilde{\sigma}}$, where $\tilde{\sigma}$ is an irreducible subrepresentation of the $\psi^{\alpha}$-descent of $\mathcal{E}_{\tau \otimes \sigma}$.

Step (3) Let $\tilde{\sigma}$ be any irreducible subrepresentation of the $\psi^{\alpha}$-descent of $\mathcal{E}_{\tau \otimes \sigma}$. $\tilde{\mathcal{E}}_{\Delta(\tau, b-1) \otimes \tilde{\sigma}}$ has a nonzero Fourier coefficient attached to the partition $\left[(2 k)^{2 b-1}\right]$.

Proof of Theorem 2.7. From the results in steps (1)-(3) above, we can see that $\mathcal{E}_{\Delta(\tau, b) \otimes \sigma}$ has a nonzero Fourier coefficient attached to the composite partition $\left[(2 k+2 m) 1^{2 k(2 b-1)}\right] \circ\left[(2 k)^{2 b-1}\right]$ (for the definition of composite partitions and the attached Fourier coefficients, we refer to [Ginzburg et al. 2003, Section 1]). Therefore, by [Jiang and Liu 2015c, Lemma 3.1] or [Ginzburg et al. 2003, Lemma 2.6], $\mathcal{E}_{\Delta(\tau, b) \otimes \sigma}$ has a nonzero Fourier coefficient attached to $\left[(2 k+2 m)(2 k)^{2 b-1}\right]$.

Before proving the above three steps, we record the following lemma which is analogous to Lemmas 4.1 and 6.1.

Lemma 7.1. Let $P_{a i}=M_{a i} N_{a i}$, with $1 \leq i \leq b$, be the parabolic subgroup of $\mathrm{Sp}_{2 a b+2 m}$ with Levi part $M_{a i} \cong \mathrm{GL}_{a i} \times \mathrm{Sp}_{a(2 b-2 i)+2 m}$. Let $\varphi$ be an arbitrary automorphic form in $\mathcal{E}_{\Delta(\tau, b) \otimes \sigma}$. Denote by $\varphi_{P_{a i}}(g)$ the constant term of $\varphi$ along $P_{a i}$. Then, for $1 \leq i \leq b$,

$$
\varphi_{P_{a i}} \in \mathcal{A}\left(N_{a i}(\mathbb{A}) M_{a i}(F) \backslash \operatorname{Sp}_{2 a b+2 m}(\mathbb{A})\right)_{\Delta(\tau, i)|\cdot|^{-(2 b-i) / 2} \otimes \mathcal{E}_{\Delta(\tau, b-i) \otimes \sigma}} .
$$

Note that when $i=b, \mathcal{E}_{\Delta(\tau, b-i) \otimes \sigma}=\sigma$.

7A. Proof of step (1). By [Ginzburg et al. 2003, Lemma 1.1], $\mathcal{E}_{\Delta(\tau, b) \otimes \sigma}$ has a nonzero Fourier coefficient attached to the partition $\left[(2 k+2 m) 1^{2 a b}\right]$ with respect to the character $\psi_{\left[(2 k+2 m) 1^{2 a b}\right], \alpha}$ if and only if the $\psi^{\alpha}$-descent

$$
\mathcal{D}_{2 k+2 m, \psi^{\alpha}}^{4 k b+2 m}\left(\mathcal{E}_{\Delta(\tau, b) \otimes \sigma}\right)
$$

of $\mathcal{E}_{\Delta(\tau, b) \otimes \sigma}$ is not identically zero, as a representation of $\widetilde{\operatorname{Sp}}_{2 k(2 b-1)}(\mathbb{A})$.

We calculate the constant term of

$$
\mathcal{F J}_{\psi_{k+m-1}^{\alpha}}^{\phi}(\xi),
$$

for $\xi \in \mathcal{E}_{\Delta(\tau, b) \otimes \sigma}$, along the parabolic subgroup

$$
\tilde{P}_{r}^{2 k(2 b-1)}(\mathbb{A})=\tilde{M}_{r}^{2 k(2 b-1)}(\mathbb{A}) N_{r}^{2 k(2 b-1)}(\mathbb{A})
$$

of $\widetilde{\mathrm{Sp}}_{2 k(2 b-1)}(\mathbb{A})$ with Levi isomorphic to $\mathrm{GL}_{r}(\mathbb{A}) \times \widetilde{\mathrm{Sp}}_{2 k}(2 b-1)-2 r(\mathbb{A})$, which is denoted by $\mathcal{C}_{N_{r}^{2 k(2 b-1)}}\left(\mathcal{F J}_{\psi_{k+m-1}^{\alpha}}^{\phi}(\xi)\right)$, where $1 \leq r \leq k(2 b-1)$. 
By [Ginzburg et al. 2011, Theorem 7.8],

$$
\begin{aligned}
\mathcal{C}_{N_{r}^{2 k(2 b-1)}}\left(\mathcal{F} \mathcal{J}_{\psi_{k+m-1}^{\alpha}}^{\phi}(\xi)\right) & \sum_{\substack{0 \leq s \leq r \\
\gamma \in P_{r-s, 1_{s}}^{1}(F) \backslash \mathrm{GL}_{r}(F)}} \int_{L(\mathbb{A})} \phi_{1}(i(\lambda)) \mathcal{F} \mathcal{J}_{\psi_{k+m-1+s}^{\alpha}}^{\phi_{2}}\left(\mathcal{C}_{N_{r-s}^{4 k b+2 m}}(\xi)\right)(\hat{\gamma} \lambda \beta) d \lambda .
\end{aligned}
$$

The notation in this formula is as follows: $N_{r-s}^{4 k b+2 m}$ is the unipotent radical of the parabolic subgroup $P_{r-s}^{4 k b+2 m}$ of $\mathrm{Sp}_{4 k b+2 m}$ with Levi isomorphic to

$$
\mathrm{GL}_{r-s} \times \mathrm{Sp}_{4 k b+2 m-2 r+2 s},
$$

and $P_{r-s, 1^{s}}^{1}$ is a subgroup of $\mathrm{GL}_{r}$ consisting of matrices of the form

$$
\left(\begin{array}{ll}
g & x \\
0 & z
\end{array}\right)
$$

with $z \in U_{s}$, the standard maximal unipotent subgroup of $\mathrm{GL}_{s}$. For $g \in \mathrm{GL}_{j}$, $j \leq 2 k b+m, \hat{g}=\operatorname{diag}\left(g, I_{4 k b+2 m-2 j}, g^{*}\right) ; L$ is a unipotent subgroup, consisting of matrices of the form

$$
\lambda=\left(\begin{array}{cc}
I_{r} & 0 \\
x & I_{k+m}
\end{array}\right)^{\wedge}
$$

$i(\lambda)$ is the last row of $x$, and

$$
\beta=\left(\begin{array}{cc}
0 & I_{r} \\
I_{k+m} & 0
\end{array}\right)^{\wedge}
$$

The Schwartz function $\phi=\phi_{1} \otimes \phi_{2}$ with $\phi_{1} \in \mathcal{S}\left(\mathbb{A}^{r}\right)$ and $\phi_{2} \in \mathcal{S}\left(\mathbb{A}^{k(2 b-1)-r}\right)$, and the function

$$
\mathcal{F J}_{\psi_{k+m-1+s}^{\alpha}}^{\phi_{2}}\left(\mathcal{C}_{N_{r-s}^{4 k b+2 m}}^{4 k}(\xi)\right)(\hat{\gamma} \lambda \beta):=\mathcal{F J}_{\psi_{k+m-1+s}^{\alpha}}^{\phi_{2}}\left(\mathcal{C}_{N_{r-s}^{4 k b+2 m}}(\rho(\hat{\gamma} \lambda \beta) \xi)\right)(I),
$$

with $\rho(\hat{\gamma} \lambda \beta)$ denoting the right translation by $\hat{\gamma} \lambda \beta$, is a composition of the restriction of $\mathcal{C}_{N_{r-k}^{2 a b+2 m}}(\rho(\hat{\gamma} \lambda \beta) \xi)$ to $\mathrm{Sp}_{4 k b+2 m-2 r+2 s}(\mathbb{A})$ with the Fourier-Jacobi coefficient

$$
\mathcal{F J}_{\psi_{k+m-1+s}^{\alpha}}^{\phi_{2}^{\alpha}}
$$

taking automorphic forms on $\mathrm{Sp}_{4 k b+2 m-2 r+2 s}(\mathbb{A})$ to those on $\widetilde{\mathrm{Sp}}_{4 k b-2 k-2 r}(\mathbb{A})$.

By the cuspidal support of $\xi, \mathcal{C}_{N_{r-s}^{4 k b+2 m}}(\xi)$ is identically zero, unless $s=r$ or $r-s=2 k l$ with $1 \leq l \leq b$. When $s=r$, since $\left[(2 k+2 m+2 r) 1^{4 k b-2 k-2 r}\right]$ is bigger than $\eta_{\mathfrak{s o}_{2 n+1}}(\mathbb{C}), \mathfrak{s p}_{2 n}(\mathbb{C})(\underline{p}(\psi))$ under the lexicographical ordering, by [Jiang and Liu 2015a, Proposition 6.4; Ginzburg et al. 2003, Lemma 1.1],

$$
\mathcal{F} \mathcal{J}_{\psi_{k+m-1+r}^{\alpha}}^{\phi_{2}^{\alpha}}(\xi)
$$

is identically zero, and hence the corresponding term is zero. When $r-s=l a$, $1 \leq l \leq b$ and $1 \leq s \leq r$, by Lemma 7.1, after restricting to $\operatorname{Sp}_{4 k(b-l)+2 m}(\mathbb{A})$, 
$\mathcal{C}_{N_{r-s}^{4 k b+2 m}}(\rho(\hat{\gamma} \lambda \beta) \xi)$ becomes a form in $\mathcal{E}_{\Delta(\tau, b-l) \otimes \sigma}$. The Arthur parameter of $\mathcal{E}_{\Delta(\tau, b-l) \otimes \sigma}$ is

$$
\psi^{\prime}=(\tau, 2 b-2 l) \boxplus \underset{i=2}{r}\left(\tau_{i}, 1\right) .
$$

Since $\left[(2 k+2 m+2 s) 1^{4 k(b-l)-2 k-2 s]}\right.$ is bigger than $\eta_{\mathfrak{s o}_{2 n^{\prime}+1}(\mathbb{C}), \mathfrak{s p}_{2 n^{\prime}}(\mathbb{C})}\left(\underline{p}\left(\psi^{\prime}\right)\right)$ under the lexicographical ordering, where $2 n^{\prime}=4 k(b-l)+2 m$, by [Jiang and Liu 2015a, Proposition 6.4; Ginzburg et al. 2003, Lemma 1.1],

$$
\mathcal{F J}_{\psi_{k+m-1+s}^{\alpha}}^{\phi_{2}}\left(\mathcal{C}_{N_{r-s}^{4 k b+2 m}}(\rho(\hat{\gamma} \lambda \beta) \xi)\right)
$$

is also identically zero, and hence the corresponding term is also zero. Therefore, the only possibilities that

$$
\mathcal{C}_{N_{r}^{2 k(2 b-1)}}\left(\mathcal{F J}_{\psi_{k+m-1}^{\alpha}}^{\phi}(\xi)\right) \neq 0
$$

are $r=2 k l, 1 \leq l \leq b$, and $s=0$. To prove that $\mathcal{F J}_{\psi_{k+m-1}^{\alpha}}^{\phi}(\xi)$ is not identically zero, we just have to show that

$$
\mathcal{C}_{N_{r}^{2 k(2 b-1)}}\left(\mathcal{F J}_{\psi_{k+m-1}^{\alpha}}^{\phi}(\xi)\right) \neq 0
$$

for some $r$.

Taking $r=2 k(b-1)$, we have

$$
\begin{aligned}
& \mathcal{C}_{N_{2 k(b-1)}^{2 k(2 b-1)}}\left(\mathcal{F J}_{\psi_{k+m-1}^{\alpha}}^{\phi}(\xi)\right) \\
& =\int_{L(\mathbb{A})} \phi_{1}(i(\lambda)) \mathcal{F} \mathcal{J}_{\psi_{k+m-1}^{\alpha}}^{\phi_{2}}\left(\mathcal{C}_{N_{2 k(b-1)}^{4 k b+2 m}}(\xi)\right)(\lambda \beta) d \lambda .
\end{aligned}
$$

By Lemma 7.1, when restricted to $\mathrm{GL}_{2 k(2 b-2)}(\mathbb{A}) \times \mathrm{Sp}_{4 k+2 m}(\mathbb{A})$,

$$
\mathcal{C}_{N_{2 k(b-1)}^{4 k b+2 m}}(\xi) \in \delta_{P_{2 k(b-1)}^{4 k b+2 m}}^{1 / 2}|\operatorname{det}|^{-\frac{b+1}{2}} \Delta(\tau, b-1) \otimes \mathcal{E}_{\tau \otimes \sigma} .
$$

It follows that the integral in (7-2) is not identically zero if and only if $\mathcal{E}_{\tau \otimes \sigma}$ has a nonzero Fourier coefficient corresponding to the partition $\left[(2 k+2 m) 1^{2 k}\right]$ with respect to the character $\psi_{\left[(2 k+2 m) 1^{2 k}\right], \alpha}$. Hence, by assumption,

$$
\mathcal{F} \mathcal{J}_{\psi_{k+m-1}^{\alpha}}^{\phi}(\xi)
$$

is not identically zero. Therefore, $\mathcal{E}_{\Delta(\tau, b) \otimes \sigma}$ has a nonzero Fourier coefficient attached to the partition $\left[(2 k+2 m) 1^{2 k(2 b-1)}\right]$ with respect to the character

$$
\psi_{\left[(2 k+2 m) 1^{2 k(2 b-1)}\right], \alpha} \cdot
$$

This completes the proof of step (1). 
7B. Proof of step (2). In order to prove the square-integrability of the descent representation

$$
\mathcal{D}_{2 k+2 m, \psi^{\alpha}}^{4 k b+2 m}\left(\mathcal{E}_{\Delta(\tau, b) \otimes \sigma}\right),
$$

we need to calculate the automorphic exponent attached to the nontrivial constant term considered in step (1) with $r=2 k(b-1)$ (for the definition of automorphic exponent, see [Mœglin and Waldspurger 1995, I.3.3]). For this, we need to consider the action of

$$
\bar{g}=\operatorname{diag}\left(g, I_{2 k}, g^{*}\right) \in \operatorname{GL}_{2 k(b-1)}(\mathbb{A}) \times{\widetilde{\operatorname{Sp}_{2 k}}}_{(\mathbb{A})} .
$$

Since $r=2 k(b-1), \beta=\left(\begin{array}{cc}0 & I_{2 k(b-1)} \\ I_{k+m} & 0\end{array}\right)^{\wedge}$. Let

$$
\tilde{g}:=\beta \operatorname{diag}\left(I_{k+m}, \bar{g}, I_{k+m}\right) \beta^{-1}=\operatorname{diag}\left(g, I_{4 k+2 m}, g^{*}\right) .
$$

Then changing variables in (5-2) via $\lambda \mapsto \tilde{g} \lambda \tilde{g}^{-1}$ will give a Jacobian $|\operatorname{det} g|^{-k-m}$. On the other hand, by [Ginzburg et al. 2011, Formula (1.4)], the action of $\bar{g}$ on $\phi_{1}$ gives $\gamma_{\psi^{-\alpha}}(\operatorname{det} g)|\operatorname{det} g|^{1 / 2}$. Therefore, $\bar{g}$ acts by $\Delta(\tau, b-1)(g)$ with character

$\delta_{P_{2 k(b-1)}^{4 k b+2 m}}^{1 / 2}|\operatorname{det} g|^{-\frac{b+1}{2}}|\operatorname{det} g|^{-k-m} \gamma_{\psi^{-\alpha}}(\operatorname{det} g)|\operatorname{det} g|^{\frac{1}{2}}$

$$
=\gamma_{\psi^{-\alpha}}(\operatorname{det} g) \delta_{P_{2 k(b-1)}^{2 k(2 b-1)}}^{1 / 2}(\bar{g})|\operatorname{det} g|^{-\frac{b}{2}} .
$$

Thus, combined with the calculation in step (1), as a function on $\operatorname{GL}_{2 k(b-1)}(\mathbb{A}) \times$ $\widetilde{\mathrm{Sp}}_{2 k}(\mathbb{A})$,

$$
\begin{aligned}
& \mathcal{C}_{N_{2 k(b-1)}^{2 k(2 b-1)}}\left(\mathcal{F} \mathcal{J}_{\psi_{k+m-1}^{\alpha}}^{\phi}(\xi)\right) \\
& \quad \in \gamma_{\psi^{-\alpha}} \delta_{P_{2 k(b-1)}^{2 k(2 b-1)}}^{1 / 2}|\operatorname{det}(\cdot)|^{-\frac{b}{2}} \Delta(\tau, b-1) \otimes \mathcal{D}_{2 k}^{4 k+2 m}\left(\mathcal{E}_{\tau \otimes \sigma}\right) .
\end{aligned}
$$

Note that by the constant formula in [op. cit., Theorem 7.8], one can easily see that

$$
\mathcal{D}_{2 k}^{4 k+2 m}\left(\mathcal{E}_{\tau \otimes \sigma}\right)
$$

is a cuspidal representation of $\widetilde{\mathrm{Sp}}_{2 k}(\mathbb{A})$. Since the cuspidal exponent of $\Delta(\tau, b-1)$ is

$$
\left\{\left(\frac{2-b}{2}, \frac{4-b}{2}, \ldots, \frac{b-2}{2}\right)\right\},
$$

the cuspidal exponent of $\mathcal{C}_{N_{2 k(b-1)}^{2 k(2 b-1)}}\left(\mathcal{F J}_{\psi_{k+m-1}^{\alpha}}^{\phi}(\xi)\right)$ is

$$
\left\{\left(\frac{2-2 b}{2}, \frac{4-2 b}{2}, \ldots,-1\right)\right\} \text {. }
$$

Hence, by the Langlands square-integrability criterion [Mœglin and Waldspurger 1995, Lemma I.4.11], the automorphic representation $\mathcal{D}_{2 k+2 m, \psi^{\alpha}}^{4 k b+2 m}\left(\mathcal{E}_{\Delta(\tau, b) \otimes \sigma}\right)$ is square-integrable. 
From (7-3), as a representation of $\mathrm{GL}_{2 k(b-1)}(\mathbb{A}) \times \widetilde{\mathrm{Sp}}_{2 k}(\mathbb{A})$, we have

$$
\begin{aligned}
& \mathcal{C}_{N_{2 k(b-1)}^{2 k(2 b-1)}}\left(\mathcal{D}_{2 k+2 m, \psi^{\alpha}}^{4 k b+2 m}\left(\mathcal{E}_{\Delta(\tau, b) \otimes \sigma}\right)\right) \\
&=\gamma_{\psi^{-\alpha}} \delta_{P_{2 k(b-1)}^{2 k(2 b-1)}}^{1 / 2}|\operatorname{det}(\cdot)|^{-\frac{b}{2}} \Delta(\tau, b-1) \otimes \mathcal{D}_{2 k, \psi^{\alpha}}^{4 k+2 m}\left(\mathcal{E}_{\tau \otimes \sigma}\right) .
\end{aligned}
$$

Therefore, using a similar argument as in Section 5B, one can see that

$$
\mathcal{D}_{2 k+2 m, \psi^{\alpha}}^{4 k b+2 m}\left(\mathcal{E}_{\Delta(\tau, b) \otimes \sigma}\right)
$$

contains an irreducible subrepresentation of the residual representation $\tilde{\mathcal{E}}_{\Delta(\tau, b-1) \otimes \tilde{\sigma}}$, where $\tilde{\sigma}$ is an irreducible generic cuspidal representation of $\widetilde{\operatorname{Sp}}_{2 k}(\mathbb{A})$ which is a subrepresentation of the $\psi^{\alpha}$-descent of $\mathcal{E}_{\tau \otimes \sigma}$, and is weakly lifting to $\tau$. Since $\tau$ is also a strong lifting of $\tilde{\sigma}$, a similar argument as in Section 5B implies that $\tilde{\mathcal{E}}_{\Delta(\tau, b-1) \otimes \tilde{\sigma}}$ is irreducible. Hence $\mathcal{D}_{2 k+2 m, \psi^{\alpha}}^{4 k b+2 m}\left(\mathcal{E}_{\Delta(\tau, b) \otimes \sigma}\right)$ must contain the whole space of residual representation $\tilde{\mathcal{E}}_{\Delta(\tau, b-1) \otimes \tilde{\sigma}}$. This completes the proof of step (2).

7C. Proof of step (3). Let $\tilde{\sigma}$ be any irreducible subrepresentation of the $\psi^{\alpha}$-descent of $\mathcal{E}_{\tau \otimes \sigma}$, then it is a generic cuspidal representation of $\widetilde{\mathrm{Sp}}_{2 k}(\mathbb{A})$. Assume that $\tilde{\sigma}$ is $\psi^{\beta}$-generic for some $\beta \in F^{*} /\left(F^{*}\right)^{2}$.

As in previous sections, we need to record the following lemma which is analogous to Lemma 5.1.

Lemma 7.2. Let $\tilde{P}_{a i}(\mathbb{A})=\tilde{M}_{a i}(\mathbb{A}) N_{a i}(\mathbb{A})$ with $1 \leq i \leq b-1$ be the parabolic subgroup of $\widetilde{\mathrm{Sp}}_{2 k(2 b-1)}(\mathbb{A})$ with Levi part

$$
\tilde{M}_{a i}(\mathbb{A}) \cong \mathrm{GL}_{a i}(\mathbb{A}) \times \widetilde{\operatorname{Sp}}_{2 k}(2 b-1-2 i)(\mathbb{A}) .
$$

Let $\varphi$ be an arbitrary automorphic form in $\tilde{\mathcal{E}}_{\Delta(\tau, b-1) \otimes \tilde{\sigma}}$. Denote by $\varphi_{P_{a i}}(g)$ the constant term of $\varphi$ along $P_{a i}$. Then, for $1 \leq i \leq b-1$,

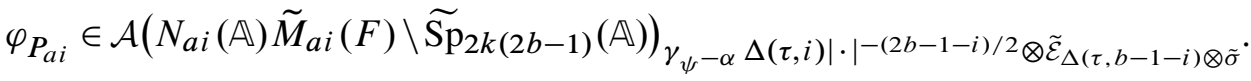
Note that when $i=b-1, \tilde{\mathcal{E}}_{\Delta(\tau, b-1-i) \otimes \tilde{\sigma}}=\tilde{\sigma}$.

First, we show that $\tilde{\mathcal{E}}_{\Delta(\tau, b-1) \otimes \tilde{\sigma}}$ has a nonzero Fourier coefficient attached to the partition $\left[(2 k) 1^{2 k(2 b-2)}\right]$ with respect to the character $\psi_{\left[(2 k) 1^{2 k(2 b-2)}\right], \beta}$. By [Ginzburg et al. 2003, Lemma 1.1], we know that $\tilde{\mathcal{E}}_{\Delta(\tau, b-1) \otimes \tilde{\sigma}}$ has a nonzero

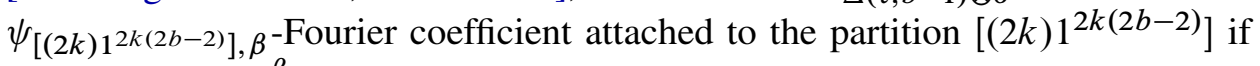
and only if the $\psi^{\beta}$-descent

$$
\tilde{\mathcal{D}}_{2 k, \psi^{\beta}}^{2 k(2 b-1)}\left(\tilde{\mathcal{E}}_{\Delta(\tau, b-1) \otimes \tilde{\sigma})}\right.
$$

of $\tilde{\mathcal{E}}_{\Delta(\tau, b-1) \otimes \tilde{\sigma}}$ is not identically zero, as a representation of $\operatorname{Sp}_{2 k(2 b-2)}(\mathbb{A})$.

Take any $\xi \in \tilde{\mathcal{E}}_{\Delta(\tau, b-1) \otimes \tilde{\sigma}}$; we will calculate the constant term of

$$
\mathcal{F J}_{\psi_{k-1}^{\beta}(\xi)}^{\phi}
$$


along $P_{r}^{2 k(2 b-2)}$, which is denoted by

$$
\mathcal{C}_{N_{r}^{2 k(2 b-2)}}\left(\mathcal{F J}_{\psi_{k-1}^{\beta}}^{\phi}(\xi)\right),
$$

where $1 \leq r \leq k(2 b-2)$. Recall that $P_{r}^{2 k(2 b-2)}=M_{r}^{2 k(2 b-2)} N_{r}^{2 k(2 b-2)}$ is the parabolic subgroup of $\mathrm{Sp}_{2 k(2 b-2)}$ with Levi subgroup isomorphic to

$$
\mathrm{GL}_{r} \times \mathrm{Sp}_{2 k(2 b-2)-2 r} .
$$

By [Ginzburg et al. 2011, Theorem 7.8],

$$
\begin{aligned}
\mathcal{C}_{N_{r}^{2 k(2 b-2)}}\left(\mathcal{F J}_{\left.\psi_{k-1}^{\beta}(\xi)\right)}^{\phi}(\xi)\right. & \sum_{\substack{0 \leq s \leq r \\
\gamma \in P_{r-s, 1^{s}}^{1}(F) \backslash \mathrm{GL}_{r}(F)}} \int_{L(\mathrm{~A})} \phi_{1}(i(\lambda)) \mathcal{F J}_{\psi_{k-1+s}^{\beta}}^{\phi_{2}}\left(\mathcal{C}_{\left.N_{r-s}^{2 k(2 b-1)}(\xi)\right)(\hat{\gamma} \lambda \eta) d \lambda .}\right.
\end{aligned}
$$

Here is the notation in the formula: $N_{r-s}^{2 k(2 b-1)}(\mathbb{A})$ is the unipotent radical of the parabolic subgroup $\tilde{P}_{r-s}^{2 k(2 b-1)}(\mathbb{A})$ of $\widetilde{\operatorname{Sp}}_{2 k(2 b-1)}(\mathbb{A})$ with Levi subgroup isomorphic to $\mathrm{GL}_{r-s}(\mathbb{A}) \times{\widetilde{\mathrm{Sp}_{2 k}}}_{(2 b-1)-2 r+2 s}(\mathbb{A}), P_{r-s, 1^{s}}^{1}$ is a subgroup of $\mathrm{GL}_{r}$ consisting of matrices of the form

$$
\left(\begin{array}{ll}
g & x \\
0 & z
\end{array}\right)
$$

with $z \in U_{s}$, the standard maximal unipotent subgroup of $\mathrm{GL}_{s}$. For $g \in \mathrm{GL}_{j}$, with $j \leq k(2 b-1), \hat{g}=\operatorname{diag}\left(g, I_{2 k(2 b-1)-2 j}, g^{*}\right), L$ is a unipotent subgroup, consisting of matrices of the form

$$
\lambda=\left(\begin{array}{cc}
I_{r} & 0 \\
x & I_{k}
\end{array}\right)^{\wedge}
$$

$i(\lambda)$ is the last row of $x$, and

$$
\eta=\left(\begin{array}{cc}
0 & I_{r} \\
I_{k} & 0
\end{array}\right)^{\wedge}
$$

The Schwartz function $\phi=\phi_{1} \otimes \phi_{2}$ with $\phi_{1} \in \mathcal{S}\left(\mathbb{A}^{r}\right)$ and $\phi_{2} \in \mathcal{S}\left(\mathbb{A}^{k(2 b-2)-r}\right)$, and the function

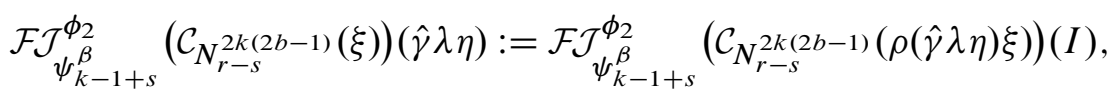

with $\rho(\hat{\gamma} \lambda \eta)$ denoting the right translation by $\hat{\gamma} \lambda \eta$, is a composition of the restriction to $\widetilde{\operatorname{Sp}}_{2 k(2 b-1)-2 r+2 s}(\mathbb{A})$ of $\mathcal{C}_{N_{r-s}^{2 a b+2 m}}(\rho(\hat{\gamma} \lambda \eta) \xi)$ with Fourier-Jacobi coefficient

$$
\mathcal{F} \mathcal{J}_{\psi_{k-1+s}^{\beta}}^{\phi_{2}}
$$

taking automorphic forms on $\widetilde{\mathrm{Sp}}_{2 k}(2 b-1)-2 r+2 s$ (A) to those on $\operatorname{Sp}_{2 k(2 b-2)-2 r}(\mathbb{A})$.

By the cuspidal support of $\xi, \mathcal{C}_{N_{r-s}^{2 k(2 b-1)}}(\xi)$ is identically zero, unless $s=r$ or $r-s=2 k l$ with $1 \leq l \leq b-1$. When $s=r$, from the structure of the unramified 
components of the residual representation $\tilde{\mathcal{E}}_{\Delta(\tau, b-1) \otimes \tilde{\sigma}}$, by [Jiang and Liu 2015a, Lemma 3.2],

$$
\mathcal{F J}_{\psi_{k-1+r}^{\beta}}^{\phi_{2}}(\xi)
$$

is identically zero, and hence the corresponding term is zero. When $r-s=2 k l$, $1 \leq l \leq b-1$ and $1 \leq s \leq r$, then by Lemma 7.2, after restricting to $\widetilde{\operatorname{Sp}}_{2 k}(2 b-1-2 l)(\mathbb{A})$, $\mathcal{C}_{N_{r-S}^{2 k(2 b-1)}}(\rho(\hat{\gamma} \lambda \eta) \xi)$ becomes a form in $\tilde{\mathcal{E}}_{\Delta(\tau, b-1-l) \otimes \tilde{\sigma}}$. From the structure of the unramified components of the residual representation $\tilde{\mathcal{E}}_{\Delta(\tau, b-1-l) \otimes \tilde{\sigma}}$, by [loc. cit.],

$$
\mathcal{F J}_{\psi_{k-1+s}^{\beta}}^{\phi_{2}}\left(\mathcal{C}_{N_{r-s}^{2 k(2 b-1)}}(\rho(\hat{\gamma} \lambda \eta) \xi)\right)
$$

is also identically zero, and hence the corresponding term is also zero. Therefore, the only possibilities that

$$
\mathcal{C}_{N_{r}^{2 k(2 b-2)}}\left(\mathcal{F J}_{\psi_{k-1}^{\beta}}^{\phi}(\xi)\right) \neq 0
$$

are $r=2 k l, 1 \leq l \leq b-1$, and $s=0$. To prove that $\mathcal{F J}_{\psi_{k-1}^{\beta}}^{\phi}(\xi)$ is not identically zero, we just have to show

for some $r$.

$$
\mathcal{C}_{N_{r}^{2 k(2 b-2)}}\left(\mathcal{F J}_{\psi_{k-1}^{\beta}}^{\phi}(\xi)\right) \neq 0
$$

Taking $r=2 k(b-1)$, we have

$$
\mathcal{C}_{N_{r}^{2 k(2 b-2)}}\left(\mathcal{F J}_{\psi_{k-1}^{\beta}}^{\phi}(\xi)\right)=\int_{L(\mathrm{~A})} \phi_{1}(i(\lambda)) \mathcal{F J}_{\psi_{k-1}^{\beta}}^{\phi_{2}}\left(\mathcal{C}_{N_{2 k(b-1)}^{2 k(2 b-1)}}(\xi)\right)(\lambda \eta) d \lambda
$$

By Lemma 7.2, when restricted to $\mathrm{GL}_{2 k(b-1)}(\mathbb{A}) \times \widetilde{\mathrm{Sp}}_{2 k}(\mathbb{A})$,

$$
\mathcal{C}_{N_{2 k(b-1)}^{2 k(2 b-1)}}(\xi) \in \delta_{P_{2 k(b-1)}^{2 k(2 b-1)}}^{1 / 2}|\operatorname{det}|^{-\frac{b}{2}} \gamma_{\psi^{-\alpha}} \Delta(\tau, b-1) \otimes \tilde{\sigma} .
$$

It is clear that the integral in (7-6) is not identically zero if and only if $\tilde{\sigma}$ is $\psi^{\beta}$ generic. Hence, by assumption,

$$
\mathcal{F} \mathcal{J}_{\psi_{k-1}^{\alpha}}^{\phi}(\xi)
$$

is not identically zero. Thus, $\tilde{\mathcal{E}}_{\Delta(\tau, b-1) \otimes \tilde{\sigma}}$ has a nonzero Fourier coefficient attached to the partition $\left[(2 k) 1^{2 k(2 b-2)}\right]$ with respect to the character $\left.\psi_{[(2 k) 12 k(2 b-2)}\right], \beta$.

Next, we show that the $\psi^{\beta}$-descent

$$
\widetilde{\mathcal{D}}_{2 k, \psi^{\beta}}^{2 k(2 b-1)}\left(\tilde{\mathcal{E}}_{\Delta(\tau, b-1) \otimes \tilde{\sigma})}\right.
$$

of $\tilde{\mathcal{E}}_{\Delta(\tau, b-1) \otimes \tilde{\sigma}}$ is square-integrable and contains the whole space of the residual representation $\mathcal{E}_{\Delta(\tau, b-1)}$ which is irreducible, as shown in [Liu 2013b, Theorem 7.1].

To prove the square-integrability of

$$
\widetilde{\mathcal{D}}_{2 k, \psi^{\beta}}^{2 k(2 b-1)}\left(\tilde{\mathcal{E}}_{\Delta(\tau, b-1) \otimes \tilde{\sigma}),}\right.
$$


we need to calculate the automorphic exponent attached to the nontrivial constant term considered above $(r=2 k(b-1))$. For this, we need to consider the action of

$$
\bar{g}=\operatorname{diag}\left(g, g^{*}\right) \in \mathrm{GL}_{2 k(b-1)}(\mathbb{A}) \times \mathrm{Sp}_{0}(\mathbb{A}) .
$$

Since $r=2 k(b-1), \eta=\left(\begin{array}{cc}0 & I_{2 k(b-1)} \\ I_{k} & 0\end{array}\right)$. Let

$$
\tilde{g}:=\eta \operatorname{diag}\left(I_{k}, \bar{g}, I_{k}\right) \eta^{-1}=\operatorname{diag}\left(g, I_{2 k}, g^{*}\right) \text {. }
$$

Then changing variables in (7-6) via $\lambda \mapsto \tilde{g} \lambda \tilde{g}^{-1}$ will give a Jacobian $|\operatorname{det} g|^{-k}$. On the other hand, by [Ginzburg et al. 2011, Formula (1.4)], the action of $\bar{g}$ on $\phi_{1}$ gives $|\operatorname{det} g|^{1 / 2}$. Therefore, $\bar{g}$ acts by $\Delta(\tau, b-1)(g)$ with character

$$
\delta_{P_{2 k(b-1)}^{2 k(2 b-1)}}^{1 / 2}|\operatorname{det} g|^{-\frac{b}{2}}|\operatorname{det} g|^{-k} \gamma_{\psi^{-\beta}}(\operatorname{det} g)|\operatorname{det} g|^{\frac{1}{2}}=\delta_{P_{2 k(b-1)}^{2 k(2 b-2)}}^{1 / 2}(\bar{g})|\operatorname{det} g|^{-\frac{b-1}{2}} .
$$

Therefore, as a function on $\mathrm{GL}_{2 k(b-1)}(\mathbb{A}) \times \mathrm{Sp}_{0}(\mathbb{A})$,

$$
\mathcal{C}_{N_{2 k(b-1)}^{2 k(2 b-2)}}\left(\mathcal{F J}_{\psi_{k-1}^{\beta}}^{\phi}(\xi)\right) \in \delta_{P_{2 k(b-1)}^{2 k(2 b-2)}}^{1 / 2}|\operatorname{det}(\cdot)|^{-\frac{b-1}{2}} \Delta(\tau, b-1) \otimes 1_{\mathrm{Sp}_{0}(\mathbb{A})} .
$$

Since the cuspidal exponent of $\Delta(\tau, b-1)$ is

$$
\left\{\left(\frac{2-b}{2}, \frac{4-b}{2}, \ldots, \frac{b-2}{2}\right)\right\},
$$

the cuspidal exponent of $\mathcal{C}_{N_{2 k(b-1)}^{2 k(2 b-2)}}\left(\mathcal{F J}_{\psi_{k-1}^{\beta}}^{\phi}(\xi)\right)$ is

$$
\left\{\left(\frac{3-2 b}{2}, \frac{5-2 b}{2}, \ldots,-\frac{1}{2}\right)\right\} \text {. }
$$

By the Langlands square-integrability criterion ([Mœglin and Waldspurger 1995, Lemma I.4.11]), the automorphic representation

$$
\tilde{\mathcal{D}}_{2 k, \psi^{\beta}}^{2 k(2 b-1)}\left(\tilde{\mathcal{E}}_{\Delta(\tau, b-1) \otimes \tilde{\sigma})}\right.
$$

is square integrable.

From (7-7), it is easy to see that as a representation of $\mathrm{GL}_{2 k(b-1)}(\mathbb{A}) \times \mathrm{Sp}_{0}(\mathbb{A})$,

$$
\begin{aligned}
& \mathcal{C}_{N_{2 k(b-1)}^{2 k(2 b-2)}\left(\widetilde { \mathcal { D } } _ { 2 k , \psi ^ { \beta } } ^ { 2 k ( 2 b - 1 ) } \left(\tilde{\mathcal{E}}_{\Delta(\tau, b-1) \otimes \tilde{\sigma}))}\right.\right.} \\
& \quad=\delta_{P_{2 k(b-1)}^{2 k(2 b-2)}}^{1 / 2}|\operatorname{det}(\cdot)|^{-\frac{b-1}{2}} \Delta(\tau, b-1) \otimes 1_{\mathrm{Sp}_{0}(\mathbb{A})} .
\end{aligned}
$$

It follows that

$$
\widetilde{\mathcal{D}}_{2 k, \psi^{\beta}}^{2 k(2 b-1)}\left(\tilde{\mathcal{E}}_{\Delta(\tau, b-1) \otimes \tilde{\sigma})}\right.
$$

has a nontrivial intersection with the space of the residual representation $\mathcal{E}_{\Delta(\tau, b-1)}$. Since by [Liu 2013b, Theorem 7.1, part (2)], $\mathcal{E}_{\Delta(\tau, b-1)}$ is irreducible,

$$
\widetilde{\mathcal{D}}_{2 k, \psi^{\beta}}^{2 k(2 b-1)}\left(\tilde{\mathcal{E}}_{\Delta(\tau, b-1) \otimes \tilde{\sigma})}\right.
$$


must contains the whole space of the residual representation $\mathcal{E}_{\Delta(\tau, b-1)}$. By [op. cit., Theorem 7.1, part (3)], the descent

$$
\tilde{\mathcal{D}}_{2 k, \psi^{\beta}}^{2 k(2 b-1)}\left(\tilde{\mathcal{E}}_{\Delta(\tau, b-1) \otimes \tilde{\sigma})}\right.
$$

is actually irreducible and equals the residual representation $\mathcal{E}_{\Delta(\tau, b-1)}$ identically.

By [op. cit., Theorem 4.2.2], we know that $\mathfrak{p}^{m}\left(\mathcal{E}_{\Delta(\tau, b-1)}\right)=\left\{\left[(2 k)^{2 b-2}\right]\right\}$. Therefore, by [Jiang and Liu 2015c, Lemma 3.1] or [Ginzburg et al. 2003, Lemma 2.6], $\tilde{\mathcal{E}}_{\Delta(\tau, b-1) \otimes \tilde{\sigma}}$ has a nonzero Fourier coefficient attached to the partition $\left[(2 k)^{2 b-1}\right]$. This completes the proof of step (3).

\section{Acknowledgments}

We would like to thank David Soudry for helpful discussion on related topics. We also would like to thank the referee for careful reading of the paper and helpful comments. This material is based upon work supported by the National Science Foundation under agreement No. DMS-1128155. Any opinions, findings and conclusions or recommendations expressed in this material are those of the authors and do not necessarily reflect the views of the National Science Foundation.

\section{References}

[Achar 2003] P. N. Achar, "An order-reversing duality map for conjugacy classes in Lusztig's canonical quotient”, Transform. Groups 8:2 (2003), 107-145. MR 2004c:20073 Zbl 1021.22003

[Arthur 2013] J. Arthur, The endoscopic classification of representations: Orthogonal and symplectic groups, American Mathematical Society Colloquium Publications 61, Amer. Math. Soc., Providence, RI, 2013. MR 3135650 Zbl 1310.22014

[Ban and Jantzen 2013] D. Ban and C. Jantzen, "The Langlands quotient theorem for finite central extensions of p-adic groups", Glas. Mat. Ser. III 48(68):2 (2013), 313-334. MR 3151110 Zbl 1304.22019

[Barbasch and Vogan 1985] D. Barbasch and D. A. Vogan, Jr., "Unipotent representations of complex semisimple groups", Ann. of Math. (2) 121:1 (1985), 41-110. MR 86i:22031 Zbl 0582.22007

[Borel and Wallach 2000] A. Borel and N. Wallach, Continuous cohomology, discrete subgroups, and representations of reductive groups, 2nd ed., Mathematical Surveys and Monographs 67, Amer. Math. Soc., Providence, RI, 2000. MR 2000j:22015 Zbl 0980.22015

[Cogdell et al. 2004] J. W. Cogdell, H. H. Kim, I. I. Piatetski-Shapiro, and F. Shahidi, "Functoriality for the classical groups", Publ. Math. Inst. Hautes Études Sci. 99:1 (2004), 163-233. MR 2006a:22010 Zbl 1090.22010

[Collingwood and McGovern 1993] D. H. Collingwood and W. M. McGovern, Nilpotent orbits in semisimple Lie algebras, Van Nostrand Reinhold, New York, 1993. MR 94j:17001 Zbl 0972.17008

[Gan et al. 2012] W. T. Gan, B. H. Gross, and D. Prasad, "Symplectic local root numbers, central critical $L$ values, and restriction problems in the representation theory of classical groups", pp. 1-109 in Sur les conjectures de Gross et Prasad, I, Astérisque 346, Société Mathématique de France, Paris, 2012. MR 3202556 Zbl 1280.22019 
[Ginzburg et al. 2003] D. Ginzburg, S. Rallis, and D. Soudry, "On Fourier coefficients of automorphic forms of symplectic groups”, Manuscripta Math. 111:1 (2003), 1-16. MR 2004g:11033 Zbl 1027.11034

[Ginzburg et al. 2004] D. Ginzburg, D. Jiang, and S. Rallis, "On the nonvanishing of the central value of the Rankin-Selberg L-functions", J. Amer. Math. Soc. 17:3 (2004), 679-722. MR 2005g:11078 Zbl 1057.11029

[Ginzburg et al. 2011] D. Ginzburg, S. Rallis, and D. Soudry, The descent map from automorphic representations of GL(n) to classical groups, World Scientific, Hackensack, NJ, 2011. MR 2012g:22020 Zbl 1233.11056

[Jiang 2014] D. Jiang, “Automorphic integral transforms for classical groups, I: Endoscopy correspondences", pp. 179-242 in Automorphic forms and related geometry: Assessing the legacy of I. I. Piatetski-Shapiro, edited by J. W. Cogdell et al., Contemp. Math. 614, Amer. Math. Soc., Providence, RI, 2014. MR 3220929 Zbl 1315.11037

[Jiang and Liu 2013] D. Jiang and B. Liu, "On Fourier coefficients of automorphic forms of GL( $n)$ ", Int. Math. Res. Not. 2013:17 (2013), 4029-4071. MR 3096918 Zbl 06438742

[Jiang and Liu 2015a] D. Jiang and B. Liu, "Arthur parameters and Fourier coefficients for automorphic forms on symplectic groups", 2015. To appear in Annales de l'Institut Fourier. arXiv 1309.6239

[Jiang and Liu 2015b] D. Jiang and B. Liu, "Fourier coefficients for automorphic forms on quasisplit classical groups", 2015. To appear in the special volume in honor of J. Cogdell. arXiv 1412.7553

[Jiang and Liu 2015c] D. Jiang and B. Liu, "On special unipotent orbits and Fourier coefficients for automorphic forms on symplectic groups", J. Number Theory 146 (2015), 343-389. MR 3267118 Zbl 06359741

[Jiang and Soudry 2003] D. Jiang and D. Soudry, "The local converse theorem for SO $(2 n+1)$ and applications", Ann. of Math. (2) 157:3 (2003), 743-806. MR 2005b:11193 Zbl 1049.11055

[Jiang et al. 2013] D. Jiang, B. Liu, and L. Zhang, "Poles of certain residual Eisenstein series of classical groups”, Pacific J. Math. 264:1 (2013), 83-123. MR 3079762 Zbl 06203663

[Jiang et al. 2015] D. Jiang, B. Liu, B. Xu, and L. Zhang, “The Jacquet-Langlands correspondence via twisted descent", Int. Math. Res. Not. (online publication October 2015). arXiv 1501.00506

[Langlands 1976] R. P. Langlands, On the functional equations satisfied by Eisenstein series, Lecture Notes in Mathematics 544, Springer, Berlin, 1976. MR 58 \#28319 Zbl 0332.10018

[Liu 2013a] B. Liu, Fourier coefficients of automorphic forms and Arthur classification, Ph.D. thesis, University of Minnesota, 2013, Available at http://search.proquest.com/docview/1419458650.

[Liu 2013b] B. Liu, "On extension of Ginzburg-Jiang-Soudry correspondence to certain automorphic forms on $\operatorname{Sp}_{4 m n}(\mathbb{A})$ and $\tilde{\mathrm{Sp}}_{4 m n \pm 2 n}(\mathbb{A})$ ", 2013. submitted. arXiv 1309.6240

[Mœglin 2008] C. Mœglin, "Formes automorphes de carré intégrable non cuspidales", Manuscripta Math. 127:4 (2008), 411-467. MR 2010i:11071 Zbl 1306.11041

[Mœglin 2011] C. Mœglin, "Image des opérateurs d'entrelacements normalisés et pôles des séries d'Eisenstein”, Adv. Math. 228:2 (2011), 1068-1134. MR 2822218 Zbl 1225.22016

[Mœglin and Waldspurger 1989] C. Mœglin and J.-L. Waldspurger, "Le spectre résiduel de GL(n)", Ann. Sci. École Norm. Sup. (4) 22:4 (1989), 605-674. MR 91b:22028 Zbl 0696.10023

[Mœglin and Waldspurger 1995] C. Mœglin and J.-L. Waldspurger, Spectral decomposition and Eisenstein series: A paraphrase of the scriptures, Cambridge Tracts in Mathematics 113, Cambridge Univ. Press, 1995. MR 97d:11083 Zbl 0846.11032

[Shahidi 2010] F. Shahidi, Eisenstein series and automorphic L-functions, American Mathematical Society Colloquium Publications 58, Amer. Math. Sci., Providence, RI, 2010. MR 2012d:11119 Zbl 1215.11054 
[Tadić 1986] M. Tadić, "Classification of unitary representations in irreducible representations of general linear group (non-Archimedean case)”, Ann. Sci. École Norm. Sup. (4) 19:3 (1986), 335-382. MR 88b:22021 Zbl 0614.22005

[Vogan 1986] D. A. Vogan, Jr., "The unitary dual of GL(n) over an Archimedean field", Invent. Math. 83:3 (1986), 449-505. MR 87i:22042 Zbl 0598.22008

Received October 21, 2014. Revised June 12, 2015.

DIHUA JIANG

UNIVERSITY OF MINNESOTA

127 VINCENT HALL

206 CHURCH ST. SE

MinNEAPOLIS, MN 55455

UNITED STATES

dhjiang@math.umn.edu

BAIYING LIU

SCHOOL OF MATHEMATICS

INSTITUTE FOR ADVANCED STUDY

EINSTEIN DRIVE

PRINCETON, NJ 08540

UNITED STATES

liu@ias.edu 


\title{
PACIFIC JOURNAL OF MATHEMATICS
}

\author{
msp.org/pjm
}

Founded in 1951 by E. F. Beckenbach (1906-1982) and F. Wolf (1904-1989)

\section{EDITORS}

Don Blasius (Managing Editor)

Department of Mathematics

University of California

Los Angeles, CA 90095-1555

blasius@math.ucla.edu

\author{
Paul Balmer \\ Department of Mathematics \\ University of California \\ Los Angeles, CA 90095-1555 \\ balmer@math.ucla.edu \\ Robert Finn \\ Department of Mathematics \\ Stanford University \\ Stanford, CA 94305-2125 \\ finn@math.stanford.edu \\ Sorin Popa \\ Department of Mathematics \\ University of California \\ Los Angeles, CA 90095-1555 \\ popa@math.ucla.edu
}

\author{
Vyjayanthi Chari \\ Department of Mathematics \\ University of California \\ Riverside, CA 92521-0135 \\ chari@math.ucr.edu \\ Kefeng Liu \\ Department of Mathematics \\ University of California \\ Los Angeles, CA 90095-1555 \\ liu@math.ucla.edu \\ Jie Qing \\ Department of Mathematics \\ University of California \\ Santa Cruz, CA 95064 \\ qing@ cats.ucsc.edu
}

\section{PRODUCTION}

Silvio Levy, Scientific Editor, production@msp.org

\section{SUPPORTING INSTITUTIONS}

ACADEMIA SINICA, TAIPEI

CALIFORNIA INST. OF TECHNOLOGY

INST. DE MATEMÁTICA PURA E APLICADA

KEIO UNIVERSITY

MATH. SCIENCES RESEARCH INSTITUTE

NEW MEXICO STATE UNIV.

OREGON STATE UNIV.

\author{
STANFORD UNIVERSITY \\ UNIV. OF BRITISH COLUMBIA \\ UNIV. OF CALIFORNIA, BERKELEY \\ UNIV. OF CALIFORNIA, DAVIS \\ UNIV. OF CALIFORNIA, LOS ANGELES \\ UNIV. OF CALIFORNIA, RIVERSIDE \\ UNIV. OF CALIFORNIA, SAN DIEGO \\ UNIV. OF CALIF., SANTA BARBARA
}

\author{
Daryl Cooper \\ Department of Mathematics \\ University of California \\ Santa Barbara, CA 93106-3080 \\ cooper@math.ucsb.edu \\ Jiang-Hua Lu \\ Department of Mathematics \\ The University of Hong Kong \\ Pokfulam Rd., Hong Kong \\ jhlu@maths.hku.hk \\ Paul Yang \\ Department of Mathematics \\ Princeton University \\ Princeton NJ 08544-1000 \\ yang@math.princeton.edu
}

These supporting institutions contribute to the cost of publication of this Journal, but they are not owners or publishers and have no responsibility for its contents or policies.

See inside back cover or msp.org/pjm for submission instructions.

The subscription price for 2016 is US $\$ 440 /$ year for the electronic version, and $\$ 600 /$ year for print and electronic.

Subscriptions, requests for back issues and changes of subscribers address should be sent to Pacific Journal of Mathematics, P.O. Box 4163, Berkeley, CA 94704-0163, U.S.A. The Pacific Journal of Mathematics is indexed by Mathematical Reviews, Zentralblatt MATH, PASCAL CNRS Index, Referativnyi Zhurnal, Current Mathematical Publications and Web of Knowledge (Science Citation Index).

The Pacific Journal of Mathematics (ISSN 0030-8730) at the University of California, c/o Department of Mathematics, 798 Evans Hall \#3840, Berkeley, CA 94720-3840, is published twelve times a year. Periodical rate postage paid at Berkeley, CA 94704, and additional mailing offices. POSTMASTER: send address changes to Pacific Journal of Mathematics, P.O. Box 4163, Berkeley, CA 94704-0163.

PJM peer review and production are managed by EditFLOW ${ }^{\circledR}$ from Mathematical Sciences Publishers.

\section{PUBLISHED BY}

\section{mathematical sciences publishers \\ nonprofit scientific publishing}

http://msp.org/

(C) 2016 Mathematical Sciences Publishers 


\title{
PACIFIC JOURNAL OF MATHEMATICS
}

\author{
Volume $281 \quad$ No. $2 \quad$ April 2016
}

The Eisenstein elements of modular symbols for level product of two 257 distinct odd primes

DEBARGHA BANERJEE and SRILAKSHMI KRISHNAMOORTHY

Primitively generated Hall algebras

ARKADY BERENSTEIN and JACOB GREENSTEIN

Generalized splines on arbitrary graphs

Simcha Gilbert, JULiAnNA TyMOCZKO and SHIRA ViEL

Good traces for not necessarily simple dimension groups

DAVID HANDELMAN

On Fourier coefficients of certain residual representations of symplectic groups

DIHUA JIANG and BAIYING LIU

On the existence of central fans of capillary surfaces

AMMAR KHANFER

Surfaces of prescribed mean curvature $H(x, y, z)$ with one-to-one central projection onto a plane

FRIEDRICH SAUVIGNY 